\title{
Creation of high-energy phonons by four-phonon processes in anisotropic phonon systems of He II
}

\author{
I.N. Adamenko ${ }^{1}$, Yu.A. Kitsenko², K.E. Nemchenko, \\ V.A. Slipko ${ }^{1}$, and A.F.G. Wyatt ${ }^{3}$ \\ ${ }^{1}$ Karazin Kharkov National University, 4, Svobody Sq., Kharkov 61077, Ukraine \\ ${ }^{2}$ Akhiezer Institute for Theoretical Physics National Science Center \\ «Kharkov Institute of Physics and Technology» National Academy of Sciences of Ukraine \\ 1 Academicheskaya Str., Kharkov 61108, Ukraine \\ ${ }^{3}$ School of Physics, University of Exeter, Exeter EX4 4QL, UK \\ E-mail: a.f.g.wyatt@exeter.ac.uk \\ Received July 31, 2006
}

\begin{abstract}
The problem of the creation of high-energy phonons (h-phonons) by a pulse of low-energy phonons (1-phonons) moving from a heater to a detector in superfluid helium, is solved. The rate of h-phonon creation is obtained and it is shown that created h-phonons occupy a much smaller solid angle in momentum space, than the 1-phonons. An analytical expression for the creation rate of h-phonon, along the symmetry axis of a pulse, are derived. It allows us to get useful approximate analytical expressions for the creation rate of h-phonons. The time dependences of the parameters which describe the 1-phonon pulse are obtained. This shows that half of the initial energy of 1-phonon pulse can be transferred into h-phonons. The results of the calculations are compared with experimental data and we show that this theory explains a number of experimental results. The value of the momentum, which separates the 1- and h-phonon subsystems, is found.
\end{abstract}

PACS: 67.70.+n Films (including physical adsorption); 68.08.-p Liquid-solid interfaces;

62.60.+v Acoustical properties of liquids.

Keywords: phonon-phonon interaction, liquid helium, anisotropic phonon system.

\section{Introduction}

The dispersion relation, which has an important role for the processes of phonon-phonon interactions in superfluid helium (He II), can be written as

$$
\varepsilon=c p(1+\psi(p))
$$

where $c$ is sound velocity, $\varepsilon$ and $p$ correspond to phonon energy and momentum, $\psi(p)$ is a function which describes the deviation of the spectrum from linearity which is small $(|\psi(p)|<<1)$, but it nevertheless completely determines the possible interactions of phonons.

When $p<p_{c}$, where $\tilde{p}_{c}=10 \mathrm{~K}$ (here and below $\left.\widetilde{p} \equiv c p / k_{B}\right)$ at zero pressure, the function $\psi>0$ and the dispersion is anomalous. Then the conservation laws of energy and momentum allow processes which do not conserve the number of phonons. The three-phonon process (3pp) is fastest of these processes and involves one phonon decaying into two or two interacting phonons combining into one.

When $p>p_{c}$ the function $\psi<0$ and the dispersion is normal. Then the decay processes are prohibited by the conservation laws of energy and momentum, and the fastest scattering is by four-phonon processes $(4 \mathrm{pp}$ or $2 \rightarrow 2)$.

The three-phonon rate $v_{3 p p}$ is much higher than the four-phonon rate $v_{4 p p}$

$$
v_{3 p p} \gg v_{4 p p}
$$

and this inequality of rates causes the phonons in superfluid helium to separate into two subsystems: a subsystem of high-energy phonons (h-phonons) with $p>p_{c}$ in which equilibrium is attained relatively slowly, and a subsystem of low-energy phonons (1-phonons) with $p<p_{c}$ in which the equilibrium occurs relatively quickly.

On the time scale of the concerned problem, the equilibrium in the subsystem of 1-phonons occurs instantly and the 
phonon distribution of this anisotropic phonon system is given by the Bose-Einstein distribution function with two parameters, temperature and drift velocity $[1,2]$.

The possibility of two-phonon subsystems with different relaxation times leads to interesting phenomena in anisotropic phonon systems of He II. Such systems are created by a heater immersed in the liquid helium, which is at such a low temperature that the thermal excitations can be neglected. When a short current pulse, of duration $t_{p}$, is given to the heater, a phonon pulse in the liquid is formed by fast three-phonon processes. This 1-phonon pulse moves in a «phonon vacuum», from the heater to the detector, with a velocity very near to $c$ [3]

In momentum space, the phonons in the pulse are mainly located in a narrow cone with a solid angle $\Omega_{p}<<1$. The transverse dimensions of this phonon pulse, near the heater, are about dimensions of the heater (typically $1 \times 1 \mathrm{~mm}$ ), and the longitudinal dimension, which is determined by the duration of current pulse, is $c t_{p}$ : typically $c t_{p}=24 \mu \mathrm{m}$.

In experiments Ref. 4 a unique phenomenon was observed: when one short current pulse was given to the heater, two-phonon pulses, well separated in time, were detected. The first comprised 1-phonons, and the second, h-phonons. From subsequent experiments (see Refs. 5, 6) it was unambiguously demonstrated that the h-phonon pulse was not injected by the heater but was created by the 1-phonon pulse during its motion from the heater to the detector. The theory of this surprising phenomenon, when a cold 1-phonon pulse with temperature close to $1 \mathrm{~K}$, creates high-energy phonons with energy $\varepsilon \geq 10 \mathrm{~K}$, was given in Refs. 7-9.

In Refs. 7-9 it was shown that 1-phonon pulse is in equilibrium due to the fast $3 \mathrm{pp}$. The h-phonons are created within the 1-phonon pulse by slow $4 p p$ with rate $v_{4 p p}$. The h-phonons have a group velocity $u_{h} \leq 189 \mathrm{~m} / \mathrm{s}$ that is smaller than the velocity of the 1-phonon pulse $c=238 \mathrm{~m} / \mathrm{s}$. The difference in these velocities and the relatively weak interaction between h- and l-phonons (see Eq. (2)) leads to the h-phonons leaving the l-phonon pulse through its rear wall and forming a pulse of noninteracting h-phonons which arrives at the detector after the 1-phonon pulse.

However the theory created in Refs. 7-9 gives no explanation for some of the phenomena observed in experiments. One of these is that the measured solid angle in momentum space occupied by the $\mathrm{h}$-phonons is much smaller than the solid angle occupied by 1-phonons. Also it was difficult to explain how the 1-phonon signal amplitude depended on heater power because the occupied solid angle in momentum space was constant during the propagation. The theory predicted that all pulses cool to about the same temperature due to the creation of h-phonons; the final temperature is where the $\mathrm{h}$-phonon creation rate is negligible. So if the temperature and occupied solid angle are the same then the signal on the detector from the pulses of different powers should be the same. But experiments showed that the 1-phonon signal amplitude increased with increasing of heater power, so it was proposed that larger solid angles were created at higher powers [10].

In this paper the exact local-equilibrium distribution function, for phonons in a pulse, is used. It allows us to obtain the angular dependence of the h-phonon creation processes, and to explain the above mentioned experimental phenomena. In Refs. 7-9 a more simple approximation of the distribution function was used, the cone approximation, in which a cone of occupied states is cut from an isotopic distribution at tempeature $T_{p}$.

Some of results presented here have been published [11]; here we expand and extend those results, and from this foundation obtain several new results. In particular, the important case of creation and decay of high-energy phonons with momentum directed along the anisotropy axis of phonon system, is investigated for the first time. For this case, an exact expression for the creation and decay rates in an anisotropic system is obtained, together with the relaxation rate of the isotropic phonon system. Hence we derive an explicit analytical expression for the rate of h-phonon creation along the anisotropy axis. We also construct an analytical approximation for the rate in range of angles where the collision integrals are not negligible. This allows us to analyse the rate dependences on all the parameters of the problem. It is shown that the h-phonon creation rate strongly depends on the momentum $p_{d}$, which separates the $\mathrm{l}$ - and h-phonon subsystems. From a comparison of the theory, developed in this paper, with experimental data, we conclude that $p_{d}=p_{c}$.

\section{The kinetic equation for the distribution function of h-phonons}

In this section we consider the four-phonon processes (4pp) which determine the creation rate of h-phonons in anisotropic and isotropic 1-phonon systems. The conservation laws of energy and momentum for $4 \mathrm{pp}$ can be written as

$$
\varepsilon_{1}+\varepsilon_{2}=\varepsilon_{3}+\varepsilon_{4}, \quad \mathbf{p}_{1}+\mathbf{p}_{2}=\mathbf{p}_{3}+\mathbf{p}_{4} .
$$

Hereinafter, the phonon with energy $\varepsilon_{1}$ and momentum $\mathbf{p}_{1}$ is the h-phonon and the other three phonons are 1-phonons. This is the type one 4pp scattering [12] and is the most important process for h-phonon creation.

The kinetic equation describing the rate of change of the distribution function, $n\left(\mathbf{p}_{1}\right) \equiv n_{1}$, due to $4 \mathrm{pp}$ can be written as

$$
\frac{d n_{1}}{d t}=N_{b}\left(\mathbf{p}_{1}\right)-N_{d}\left(\mathbf{p}_{1}\right)
$$


where $N_{b}\left(\mathbf{p}_{1}\right)$ and $N_{d}\left(\mathbf{p}_{1}\right)$ are, respectively, the rates of increasing and decreasing numbers of h-phonons in the state with momentum $\mathbf{p}_{1}$, due to collisions. $N_{b, d}\left(\mathbf{p}_{1}\right)$ can be written as

$$
\begin{gathered}
N_{b, d}\left(\mathbf{p}_{1}\right)= \\
=\int W\left(\mathbf{p}_{1}, \mathbf{p}_{2} ; \mathbf{p}_{3}, \mathbf{p}_{4}\right) \delta\left(\mathbf{p}_{\Sigma}\right) \delta\left(\varepsilon_{\Sigma}\right) n_{b, d} d^{3} p_{2} d^{3} p_{3} d^{3} p_{4} .
\end{gathered}
$$

Here $W\left(\mathbf{p}_{1}, \mathbf{p}_{2} ; \mathbf{p}_{3}, \mathbf{p}_{4}\right)$ defines the probability density of the process ( 3 ), the $\delta$-functions correspond to the conservation of energy and momentum; $\varepsilon_{\Sigma}=\varepsilon_{1}+\varepsilon_{2}-\varepsilon_{3}-\varepsilon_{4}$ and $\mathbf{p}_{\Sigma}=\mathbf{p}_{1}+\mathbf{p}_{2}-\mathbf{p}_{3}-\mathbf{p}_{4}$. The factors $n_{b, d}$ are

$$
\begin{aligned}
& n_{b}=n_{3}^{(0)} n_{4}^{(0)}\left(1+n_{2}^{(0)}\right)\left(1+n_{1}\right), \\
& n_{d}=n_{1} n_{2}^{(0)}\left(1+n_{3}^{(0)}\right)\left(1+n_{4}^{(0)}\right),
\end{aligned}
$$

where $n_{l}^{(0)} \equiv n^{(0)}\left(\mathbf{p}_{l}\right)$ is the equilibrium distribution function of 1-phonons which are considered to be instantaneously in equilibrium due to three-phonon processes.

The equilibrium distribution function of 1-phonons as intoduced in Refs. 1, 2 can be written as

$$
n^{(0)}\left(\mathbf{p}_{l}\right)=\left\{\exp \left(\frac{\varepsilon_{l}-\mathbf{p}_{l} \mathbf{u}}{k_{B} T}\right)-1\right\}^{-1} .
$$

The drift velocity $\mathbf{u}$ can be written as

$$
\mathbf{u}=\mathbf{N} c(1-\chi),
$$

where $\mathbf{N}$ is the unit vector directed along the direction of the total momentum of the 1-phonon system; this defines the anisotropy axis of the phonon system. This equation defines $\chi$, the anisotropy parameter. In weakly anisotropic systems, $\chi$ is close to unity. For strongly anisotropic systems, which corresponds to the experiments [4-6,13,14], $\chi<<1$.

In all further calculations it will be more convenient to us to use the expression

$$
n^{(0)}\left(p_{l}, \zeta_{l}\right)=\left\{\exp \left(\frac{c p_{l}}{k_{B} T}\left(\psi_{l}+\chi+\zeta_{l}-\zeta_{l} \chi\right)\right)-1\right\}^{-1}
$$

where $\zeta_{l}=1-\mathbf{p}_{l} \mathbf{N} / p_{l}$. Equation (9) was obtained by substitution of Eqs. (1) and (8) into (7).

In an earlier description of anisotropic phonon systems we used the approximate distribution function (see Refs. 7-9) which can be written as

$$
n_{p}^{(0)}\left(\mathbf{p}_{l}\right)=\eta\left(\theta_{p}-\theta_{l}\right)\left\{\exp \left(\varepsilon_{l} / k_{B} T_{p}\right)-1\right\}^{-1} .
$$

This distribution function has a simple physical meaning and includes all the parameters of anisotropic phonon systems: temperature $T_{p}$ and the anisotropy which is defined by the cone angle $\theta_{p}$. The values of $T_{p}$ and $\theta_{p}$ can be estimated from the experimental data. However the description of the creation of h-phonons, at any angle to the anisotropy axis (see, for example, [1,2]), can only be made using the exact local-equilibrium distribution function (7) for the 1-phonons. As that is the purpose of this paper, we shall use only the parameters $\chi$ and $T$ from now on. The relationships between the parameters $\chi, T$ and $\theta_{p}, T_{p}$ come from the equating energy and momentum in the two descriptions, and were obtained in Refs. 1, 2.

The interaction of phonons in superfluid helium is described by the Landau Hamiltonian (see, for example, [15]), which we write as

$$
\hat{H}_{\mathrm{ph}}=\hat{H}_{0}+\hat{V}_{3}+\hat{V}_{4} .
$$

Here $\hat{H}_{0}$ is the Hamiltonian of noninteracting phonons. The terms $\hat{V}_{3}$ and $\hat{V}_{4}$ describe the interaction of phonons to the third and fourth orders of small deviations of a system from an equilibrium state, respectively.

The probability density of four-phonon process following Refs. 9, 15, 16, can be written as

$$
W\left(\mathbf{p}_{1}, \mathbf{p}_{2} ; \mathbf{p}_{3}, \mathbf{p}_{4}\right)=\frac{2 \pi}{\hbar} V^{2}\left|H_{f i}\right|^{2} \frac{1}{(2 \pi \hbar)^{6}} \text {. }
$$

Here $V$ is the volume of system and $H_{f i}$ is the amplitude of four-phonon process which is obtained by second order perturbation theory on $\hat{V}_{3}$ and first order perturbation theory on $\hat{V}_{4}$ with a help of standard procedures (see, for example, $[9,15-18])$. Hence

$H_{f i}=\sum_{Q} \frac{\left\langle\mathbf{p}_{3}, \mathbf{p}_{4}\left|\hat{V}_{3}\right| \mathbf{Q}\right\rangle\left\langle\mathbf{Q}\left|\hat{V}_{3}\right| \mathbf{p}_{1}, \mathbf{p}_{2}\right\rangle}{E_{i}-E_{\mathbf{Q}}}+\left\langle\mathbf{p}_{3}, \mathbf{p}_{4}\left|\hat{V}_{4}\right| \mathbf{p}_{1}, \mathbf{p}_{2}\right\rangle$,

where $\mathbf{Q}$ is an intermediate state with energy $E_{\mathbf{Q}}$, and $E_{i}$ is the energy of initial state.

The interaction of phonons with momenta $\mathbf{p}_{1}$ and $\mathbf{p}_{2}$ to create phonons with momenta $\mathbf{p}_{3}$ and $\mathbf{p}_{4}$, has six intermediate states I-VI in which phonons have momenta

$$
\begin{aligned}
& \text { I. } \mathbf{p}_{1}+\mathbf{p}_{2} ; \text { II. } \mathbf{p}_{2}, \mathbf{p}_{3}, \mathbf{p}_{1}-\mathbf{p}_{3} ; \text { III. } \mathbf{p}_{2}, \mathbf{p}_{4}, \mathbf{p}_{1}-\mathbf{p}_{4} ; \\
& \text { IV. } \mathbf{p}_{1}, \mathbf{p}_{3}, \mathbf{p}_{2}-\mathbf{p}_{3} ; \text { V. } \mathbf{p}_{1}, \mathbf{p}_{4}, \mathbf{p}_{2}-\mathbf{p}_{4} ; \\
& \text { VI. } \mathbf{p}_{1}, \mathbf{p}_{2}, \mathbf{p}_{3}, \mathbf{p}_{4},-\mathbf{p}_{1}-\mathbf{p}_{2} \text {. }
\end{aligned}
$$

From expressions (13) and (14) we obtain

$$
H_{f i}=\frac{\sqrt{p_{1} p_{2} p_{3} p_{4}}}{8 \rho V} M_{\Sigma},
$$

where $\rho=145 \mathrm{~kg} / \mathrm{m}^{3}$ is the density of He II,

$$
M_{\Sigma}=M^{(1)}+M_{13}^{(2)}+M_{14}^{(3)}+M_{23}^{(3)}+M_{24}^{(3)}+M^{(5)}+M_{4}
$$

is the matrix element for 4pp, which consists of seven terms, six of which correspond to the six intermediate states (14), and the seventh, to first order of perturbation theory on $\hat{V}_{4}$. 
The superscripts show the number of phonons in the intermediate state. We can write the matrix elements as

$$
\begin{aligned}
M^{(1)}= & \frac{\varepsilon_{1+2}}{\varepsilon_{1}+\varepsilon_{2}-\varepsilon_{1+2}}\left(2 u-1+\mathbf{n}_{1} \mathbf{n}_{2}+\mathbf{n}_{1} \mathbf{n}_{1+2}+\mathbf{n}_{2} \mathbf{n}_{1+2}\right) \times \\
& \times\left(2 u-1+\mathbf{n}_{3} \mathbf{n}_{4}+\mathbf{n}_{3} \mathbf{n}_{3+4}+\mathbf{n}_{4} \mathbf{n}_{3+4}\right), \\
M^{(5)}=- & \frac{\varepsilon_{1+2}}{\varepsilon_{1}+\varepsilon_{2}+\varepsilon_{1+2}}\left(2 u-1+\mathbf{n}_{1} \mathbf{n}_{2}-\mathbf{n}_{1} \mathbf{n}_{1+2}-\mathbf{n}_{2} \mathbf{n}_{1+2}\right) \times \\
& \times\left(2 u-1+\mathbf{n}_{3} \mathbf{n}_{4}-\mathbf{n}_{3} \mathbf{n}_{3+4}-\mathbf{n}_{4} \mathbf{n}_{3+4}\right), \\
M_{13}^{(3)}= & \frac{\varepsilon_{1-3}}{\varepsilon_{1}-\varepsilon_{3}-\varepsilon_{1-3}}\left(2 u-1+\mathbf{n}_{1} \mathbf{n}_{3}+\mathbf{n}_{1} \mathbf{n}_{1-3}+\mathbf{n}_{3} \mathbf{n}_{1-3}\right) \times \\
& \times\left(2 u-1+\mathbf{n}_{2} \mathbf{n}_{4}+\mathbf{n}_{2} \mathbf{n}_{1-3}+\mathbf{n}_{4} \mathbf{n}_{1-3}\right) .
\end{aligned}
$$

Other terms of expression (16), i.e., $M_{23}^{(3)}, M_{14}^{(3)}, M_{24}^{(3)}$ can be obtained from $M_{13}^{(3)}$ by permuting the corresponding subscripts. Here $\mathbf{n}_{i}=\mathbf{p}_{i} / p_{i}, \quad \varepsilon_{i}=\varepsilon\left(\mathbf{p}_{i}\right)$, $u=(\rho / c) \times(\partial c / \partial \rho)=2.84$ is the Grüneisen constant and $w=\left(\rho^{2} / c\right)\left(\partial^{2} c / \partial \rho^{2}\right)=0.188$. The matrix elements are given in detail in the Appendix A.

We note, that first three terms of Eq. (16) are resonant: when $\psi(p)=0$ their denominators can vanish, which leads to an essential divergence of the matrix element. These three terms give the main contribution to Eq. (16). The rest of the terms give small contributions of the same order of magnitude.

We define the rate of the $4 \mathrm{pp}$ scattering by

$$
\begin{gathered}
v_{1}\left(\mathbf{p}_{1}\right)=\int d^{3} p_{2} d^{3} p_{3} d^{3} p_{4} \times \\
\times W\left(\mathbf{p}_{1}, \mathbf{p}_{2} ; \mathbf{p}_{3}, \mathbf{p}_{4}\right) \delta\left(\mathbf{p}_{\Sigma}\right) \delta\left(\varepsilon_{\Sigma}\right) n_{2}^{(0)}\left(1+n_{3}^{(0)}\right)\left(1+n_{4}^{(0)}\right) .
\end{gathered}
$$

Then from Eq. (5), the expression for $N_{d}$ can be written as

$$
N_{d}=n_{1} v_{1} \text {. }
$$

For $N_{b}$ we use the equality

$n_{3}^{(0)} n_{4}^{(0)}\left(1+n_{2}^{(0)}\right)=\exp \left(-\frac{\varepsilon_{1}-\mathbf{p}_{1} \mathbf{u}}{k_{B} T}\right) n_{2}^{(0)}\left(1+n_{3}^{(0)}\right)\left(1+n_{4}^{(0)}\right)$,

which follows from the Eqs. (3) and (7). Then we can write

$$
N_{b}=\exp \left(-\frac{\varepsilon_{1}-\mathbf{p}_{1} \mathbf{u}}{k_{B} T}\right)\left(1+n_{1}\right) v_{1} .
$$

For short pulses that were used in experiments $[13,14], n_{1}$ is much less than the unity. (We shall only consider short pulses from now on.) Therefore the relation

$$
N_{b}^{(\mathrm{sh})}=\exp \left(-\frac{\varepsilon_{1}-\mathbf{p}_{1} \mathbf{u}}{k_{B} T}\right) v_{1}
$$

is always satisfied in short pulses.

To see the important role of $\zeta_{1}$ in determining the creation rate of h-phonons, we rewrite the exponent in (25):

$$
-\frac{\left(\varepsilon_{1}-\mathbf{p}_{1} \mathbf{u}\right)}{k_{B} T}=-\frac{c p_{1}}{k_{B} T}\left(\psi_{1}+\chi+\zeta_{1}-\zeta_{1} \chi\right) .
$$

For h-phonons $\psi_{1}$ is small and negative: when $10 \mathrm{~K}<\widetilde{p}_{1}<11 \mathrm{~K}$ then $0>\psi>-0.023$. For the anisotropic phonon systems studied experimentally, the energy densities in the liquid helium and the angular distribution of the 1-phonons are consistent with $\chi \sim 0.02$ and temperature $T \lesssim 0.04 \mathrm{~K}$. These values of $T=0.041 \mathrm{~K}$ and $\chi=0.02$ in the distribution function (9) correspond to the same energy density and momentum density as the values $T_{p}=1 \mathrm{~K}$ and $\theta_{p}=12^{\circ}$ in the cone distribution function (10). Although $\chi$ and $\psi$ are small, $\zeta_{1}$ is not necessarily small; it can vary from 0 , when $p_{1}$ is along the anisotropy axis, to 2 , when $p_{1}$ is antiparallel to the anisotropy axis. When $\zeta_{1}$ is nearly zero then the modulus of the exponent is not large, and the exponential term in (25) is not so small, and the creation rate is not small. However when $\zeta_{1}>\chi$ and $|\psi|$, then the exponent is large and negative, and the exponential term in (25) is very small, which makes the creation rate negligible.

In order to calculate the creation rate $N_{b}$ and the decay rate $N_{d}$, which appear in the kinetic equation (4), we must find the rate $v_{1}$ which is done in the next section.

\section{The rate of h-phonons creation and decay in the anisotropic l-phonon system}

\subsection{The general expression for the rate $v_{1}$}

To derive the general expression for the rate $v_{1}$, we rewrite the expression (21), taking into account (12)-(15), in spherical coordinates

$v_{1}=\frac{p_{1}}{2^{11} \pi^{5} \hbar^{7} \rho^{2}} \int d p_{2} d \varphi_{2} d \zeta_{2} d p_{3} d \varphi_{3} d \zeta_{3} d p_{4} d \varphi_{4} d \zeta_{4} p_{2}^{3} p_{3}^{3} p_{4}^{3} \times$

$$
\times M_{\Sigma}^{2} \delta\left(\mathbf{p}_{\Sigma}\right) \delta\left(\varepsilon_{\Sigma}\right) n_{2}^{(0)}\left(1+n_{3}^{(0)}\right)\left(1+n_{4}^{(0)}\right),
$$

where $\zeta_{i}=1-\mathbf{p}_{i} \mathbf{N} / p_{i}$.

Without any restriction on generality, we can choose the angle $\varphi_{1}$ as the computing origin of angles $\varphi_{i}$. In this case the $\delta$-functions can be written as

$$
\begin{aligned}
\delta\left(\mathbf{p}_{\Sigma}\right)=\delta\left(p_{1 \perp}\right. & \left.+p_{2 \perp} \cos \varphi_{2}-p_{3 \perp} \cos \varphi_{3}-p_{4 \perp} \cos \varphi_{4}\right) \times \\
\times \delta\left(p_{2 \perp}\right. & \left.\sin \varphi_{2}-p_{3 \perp} \sin \varphi_{3}-p_{4 \perp} \sin \varphi_{4}\right) \times \\
& \times \delta\left(p_{1 \|}+p_{2 \|}-p_{3 \|}-p_{4 \|}\right) .
\end{aligned}
$$


$\delta\left(\varepsilon_{\Sigma}\right)=\frac{1}{c} \delta\left(p_{1}+p_{2}-p_{3}-p_{4}-\phi\right)$,

where $p_{i \perp}=p_{i} \sqrt{2 \zeta_{i}-\zeta_{i}^{2}}, p_{i \|}=p_{i}\left(1-\zeta_{i}\right)$,

$$
\phi=p_{3} \psi_{3}+p_{4} \psi_{4}-p_{1} \psi_{1}-p_{2} \psi_{2} .
$$

We note that the value $\phi$, when $p_{1}$ is an h-phonon, is always positive $(\phi>0)$.

In order to make the integration with respect to $\varphi_{3}$ and $\varphi_{4}$ we introduce new variables

$$
\begin{gathered}
X=p_{3 \perp} \cos \varphi_{3}+p_{4 \perp} \cos \varphi_{4}, \\
Y=p_{3 \perp} \sin \varphi_{3}+p_{4 \perp} \sin \varphi_{4} .
\end{gathered}
$$

Then the expression (27) can be written as $v_{1}=\frac{p_{1}}{2^{10} \pi^{5} \hbar^{7} \rho^{2}} \int \frac{p_{2}^{3} p_{3}^{3} p_{4}^{3} d p_{2} d p_{3} d p_{4} d \zeta_{2} d \zeta_{3} d \zeta_{4} d \varphi_{2} d X d Y}{\sqrt{4 p_{3 \perp}^{2} p_{4 \perp}^{2}-\left(X^{2}+Y^{2}-p_{3 \perp}^{2}-p_{4 \perp}^{2}\right)^{2}}} \times$

$$
\times M_{\Sigma}^{2} \delta\left(\mathbf{p}_{\Sigma}\right) \delta\left(\varepsilon_{\Sigma}\right) n_{2}^{(0)}\left(1+n_{3}^{(0)}\right)\left(1+n_{4}^{(0)}\right) .
$$

Hereinafter the integration is made so that the radicands are not negative.

As $M_{\Sigma}$ depends on $\cos \varphi_{3}$ and $\cos \varphi_{4}$, to make the integration it is necessary to solve the system of the equations

$$
\left\{\begin{array}{l}
p_{1 \perp}+p_{2 \perp} \cos \varphi_{2}-p_{3 \perp} \cos \varphi_{3}-p_{4 \perp} \cos \varphi_{4}=0 \\
p_{2 \perp} \sin \varphi_{2}-p_{3 \perp} \sin \varphi_{3}-p_{4 \perp} \sin \varphi_{4}=0
\end{array}\right.
$$

with respect to $\cos \varphi_{3}$ and $\cos \varphi_{4}$.

The system of equations (33) has two solutions which can be written as

$\cos \varphi_{3}^{( \pm)}=\frac{\left(p_{1 \perp}+p_{2 \perp} \cos \varphi_{2}\right)\left(A+p_{3 \perp}^{2}-p_{4 \perp}^{2}\right) \pm p_{2 \perp} \sin \varphi_{2} \sqrt{R}}{2 A p_{3 \perp}}$,

$\cos \varphi_{4}^{( \pm)}=\frac{\left(p_{1 \perp}+p_{2 \perp} \cos \varphi_{2}\right)\left(A-p_{3 \perp}^{2}+p_{4 \perp}^{2}\right) \mp p_{2 \perp} \sin \varphi_{2} \sqrt{R}}{2 A p_{4 \perp}}$,

where

$$
A=p_{1 \perp}^{2}+p_{2 \perp}^{2}+2 p_{1 \perp} p_{2 \perp} \cos \varphi_{2},
$$

$R=4 p_{3 \perp}^{2} p_{4 \perp}^{2}-\left(p_{1 \perp}^{2}+p_{2 \perp}^{2}-p_{3 \perp}^{2}-p_{4 \perp}^{2}+2 p_{1 \perp} p_{2 \perp} \cos \varphi_{2}\right)^{2}$.

At first we integrate with respect to $X$ and $Y$, and then with respect to $p_{4}$ and $\zeta_{4}$ with the help of the $\delta$-functions. As a result we have

$$
v_{1}=K p_{1} \int d p_{2} d p_{3} d \zeta_{2} d \zeta_{3} d \varphi_{2} \frac{p_{2}^{3} p_{3}^{3} p_{4}^{2}}{\sqrt{R}} \times
$$

$$
\times\left\{M_{(+)}^{2}+M_{(-)}^{2}\right\} n_{2}^{(0)}\left(1+n_{3}^{(0)}\right)\left(1+n_{4}^{(0)}\right),
$$

where

$$
K=\frac{1}{2^{10} \pi^{5} \hbar^{7} \rho^{2} c},
$$

$$
\begin{gathered}
M_{( \pm)}=M_{\Sigma}\left(\cos \varphi_{3}=\cos \varphi_{3}^{( \pm)}, \cos \varphi_{4}=\cos \varphi_{4}^{( \pm)}\right), \\
p_{4}=p_{1}+p_{2}-p_{3}-\phi, \\
\zeta_{4}=\frac{p_{1} \zeta_{1}+p_{2} \zeta_{2}-p_{3} \zeta_{3}-\phi}{p_{4}} .
\end{gathered}
$$

Further integration cannot be precisely made analytically because of the complicated integrand expression. Therefore we first consider the important case of $\zeta_{1}=0$ and secondly present some dependences of the rate $v_{1}$ obtained from Eq. (38).

\subsection{The rate $v_{1}$ for h-phonons moving along the anisotropy axis}

This case is important as it is possible to obtain an explicit analytical expression for $v_{1}$. In Ref. 9 the rates of four-phonon processes were calculated when $\zeta_{1}=0$ using the approximate cone distribution function (10).

When $\zeta_{1}=0$, the expression (38) becomes much simpler as firstly the dependence of matrix element on $\varphi_{3}$ and $\varphi_{4}$ vanishes, and secondly the dependence of the integrand expression on $\varphi_{2}$ disappears. So the integration $\operatorname{over} \varphi_{2}$ can be easily made analytically. Hence we find

$$
\begin{array}{r}
v_{1}=2 \pi K p_{1} \int \frac{d p_{2} d p_{3} d \zeta_{2} d \zeta_{3}}{\sqrt{\left(\zeta_{3+}^{\zeta_{1}=0}-\zeta_{3}\right)\left(\zeta_{3}-\zeta_{3-}^{\zeta_{1}=0}\right)}} \times \\
\times\left(1-\frac{2 p_{1} p_{2} \zeta_{2}}{\left(p_{1}+p_{2}\right)^{2}}\right)^{-\frac{1}{2}} \times \\
\times \frac{p_{2}^{3} p_{3}^{2} p_{4}^{2}}{p_{1}+p_{2}}\left(M_{\Sigma}^{\zeta_{1}=0}\right)^{2} n_{2}^{(0)}\left(1+n_{3}^{(0)}\right)\left(1+n_{4}^{(0)}\right),
\end{array}
$$

where

$$
M_{\Sigma}^{\zeta_{1}=0}=M_{(+)}\left(\zeta_{1}=0\right)=M_{(-)}\left(\zeta_{1}=0\right)
$$

$$
=\frac{b^{\zeta_{1}=0} \pm 8 p_{1} p_{2}^{2} p_{3} \sqrt{\zeta_{2}\left(2-\zeta_{2}\right)\left(\zeta_{2}-\zeta_{2 \min }\right)\left(\zeta_{2 \max }-\zeta_{2}\right)}}{2\left|a^{\zeta_{1}=0}\right|} .
$$

Here 


$$
\begin{gathered}
b^{\zeta_{1}=0}=-8 p_{1} p_{2}^{2} p_{3} \zeta_{2}^{2}+4 p_{2} p_{3}\left(2 p_{1} p_{4}+2 p_{2} p_{3}+2 p_{1} \phi+\right. \\
\left.+2 p_{4} \phi+\phi^{2}\right) \zeta_{2}-4 p_{3} \phi\left(p_{1}+p_{2}\right)\left(2 p_{4}+\phi\right) \\
a^{\zeta_{1}=0}=-4 p_{3}^{2}\left(p_{1}+p_{2}\right)^{2}\left(1-\frac{2 p_{1} p_{2} \zeta_{2}}{\left(p_{1}+p_{2}\right)^{2}}\right) \\
\zeta_{2 \min }=\frac{\left(2 p_{3}+2 p_{4}+\phi\right) \phi}{2 p_{1} p_{2}} \\
\zeta_{2 \max }=\frac{\left(2 p_{3}+\phi\right)\left(2 p_{4}+\phi\right)}{2 p_{1} p_{2}}
\end{gathered}
$$

We note that Eq. (43) for $v_{1}$ is very important as it not only describes the rate of h-phonon creation at $\theta_{1}=0$ but also gives the relaxation in isotropic phonon system, i.e., when $\chi=1$, because in the isotropic case there is no dependence on $\theta_{1}$ and every direction is identical to $\theta_{1}=0$.

Following Ref. 9, we can replace $M_{\Sigma}^{\zeta_{1}=0}$ with $M$ which can be written as

$$
M=4(u+1)^{2}\left(m_{2}-m_{3}-m_{4}\right) \equiv 4(u+1)^{2} m .
$$

Here

$$
\begin{aligned}
& m_{2}=\frac{\left(p_{1}+p_{2}\right)^{2}}{p_{1} p_{2}} \frac{1}{\zeta_{2}+\kappa_{2}}, \\
& m_{3}=\frac{\left(p_{1}-p_{3}\right)^{2}}{p_{1} p_{3}} \frac{1}{\zeta_{3}+\kappa_{3}}, \\
& m_{4}=\frac{\left(p_{1}-p_{4}\right)^{2}}{p_{1} p_{4}} \frac{1}{\zeta_{4}+\kappa_{4}},
\end{aligned}
$$

where $\kappa_{i}$ are functions dependent on the dispersion:

$$
\begin{gathered}
\kappa_{2}=\frac{p_{1}+p_{2}}{p_{1} p_{2}}\left(f_{1}+f_{2}-f_{1+2}\right), \\
\kappa_{3}=\frac{p_{1}-p_{3}}{p_{1} p_{3}}\left(f_{3}+f_{1-3}-f_{1}\right), \\
\kappa_{4}=\frac{p_{1}-p_{4}}{p_{1} p_{4}}\left(f_{4}+f_{1-4}-f_{1}\right) \text { and } f_{i}=p_{i} \psi\left(p_{i}\right) .
\end{gathered}
$$

For all further calculations it is necessary to have an analytical approximation for the function $\psi(p)$. In this section and further on, we shall use the approximation of function $\psi(p)$, which was obtained in [9]

$$
\psi(p)=\left\{\begin{array}{l}
\alpha \frac{c-u_{c}}{2 c} \frac{p^{2}}{p_{c}^{2}}\left(1-\sigma \frac{p^{2}}{p_{c}^{2}}\right), p \leq p_{f}, \\
-\frac{p-p_{c}}{p}\left[\frac{c-u_{c}}{c}-\frac{u_{c}^{\prime}}{2 c}\left(p-p_{c}\right)\right], \quad p_{f} \leq p \leq p_{\psi} .
\end{array}\right.
$$

Here $\tilde{p}_{c}=10 \mathrm{~K}, \tilde{p}_{f}=8.26 \mathrm{~K}, \tilde{p}_{\psi}=27 \mathrm{~K}, \alpha=1.76$, $\sigma=1.13, \quad u_{c}=\partial \varepsilon /\left.\partial p\right|_{p=p_{c}}=189 \mathrm{~m} / \mathrm{s}, \quad k_{B} u_{c}^{\prime} / c=$ $=\left.\left(k_{B} / c\right)\left(\partial^{2} \varepsilon / \partial p^{2}\right)\right|_{p=p_{c}}=-19.8 \mathrm{~m} /(\mathrm{s} \cdot \mathrm{K})$.

Further integration can be made by replacing, in the slowly varying functions of momenta and angles in the integrand of Eq. (43), the momenta and angles by their typical values. As a result we have

$$
\begin{aligned}
& v_{1}=K_{0} T^{2} \exp \left(\frac{c p_{1} \psi_{1}}{k_{B} T}\right) \bar{m}^{2}\left(p_{3}=p_{c}, p_{2}=p_{c}-\frac{p_{1}}{2}\right) \times \\
& \times\left(\frac{p_{1} p_{c}}{p_{c}+p_{1} / 2}\right)^{2} \frac{12+\beta\left(p_{1}-p_{c}\right)\left(6+\beta\left(p_{1}-p_{c}\right)\right)}{\beta^{5}} .
\end{aligned}
$$

Here

$$
\begin{gathered}
K_{0}=\frac{(u+1)^{4}}{2^{4} \pi^{3} \hbar^{7} \rho^{2}\left(c-u_{c}\right)}\left(\frac{k_{B}}{c}\right)^{2}, \\
\bar{m}=m\left(\zeta_{2}=\frac{p_{1}+p_{2}}{p_{1} p_{2}} \phi, \zeta_{3,4}=\frac{p_{2} \phi}{p_{1}\left(p_{1}+p_{2}\right)}\right), \\
\beta=\frac{c\left(\psi_{2 t}+\chi-\psi_{1}\right)}{k_{B} T}, \text { where } \psi_{2 t}=0.02 .
\end{gathered}
$$

The result (57) allows us to analyze the dependences of the rate $v_{1}$ on $p_{1}, T, \chi$ and the parameters of liquid helium. So for example, the fast decrease of $v_{1}$ with increasing $p_{1}$ is mainly caused by the exponent. We see from (57) that the rate is proportional to the minus one third power of the dispersion as $\psi \rightarrow 0$. Moreover the form of result (57) will be used below when obtaining an approximation for the rate $v_{1}$ in the range of not too-large $\zeta_{1}$, which is mainly realized in a pulse. We note that Eq. (57) is in good agreement with the results of exact numerical calculation of the rates from Eq. (43) near $T=0.041 \mathrm{~K}$ and $\chi=0.02$. At other values of $\chi$ and $T$ the numerical agreement with the rates calculated from Eq. (43) are not so good, but the dependences are qualitatively the same.

We note, that in obtaining the result (57), we used the fact that the main contribution, in integration over $p_{3}$, is due to phonons with momenta close to $p_{c}$, which we have assumed separates the 1- and h-phonon subsystems. This leads to the conclusion that the rate of h-phonon creation strongly depends on the numerical value of momentum which separates the $1-$ and h-phonon subsystems. The possibility that this momentum, $p_{d}$, which separates the 1and h-phonon subsystems, is not equal to $p_{c}$ will be discussed in section 6 .

\subsection{Dependence of the rate $v_{1}$ on all parameters}

The dependences of $v_{1}$ on $p_{1}, \zeta_{1}, T$ and $\chi$, can only be obtained by numerical integration of Eq. (38). The results of this are shown in Fig. 1: in Fig. 1, $a$ is shown the angular 

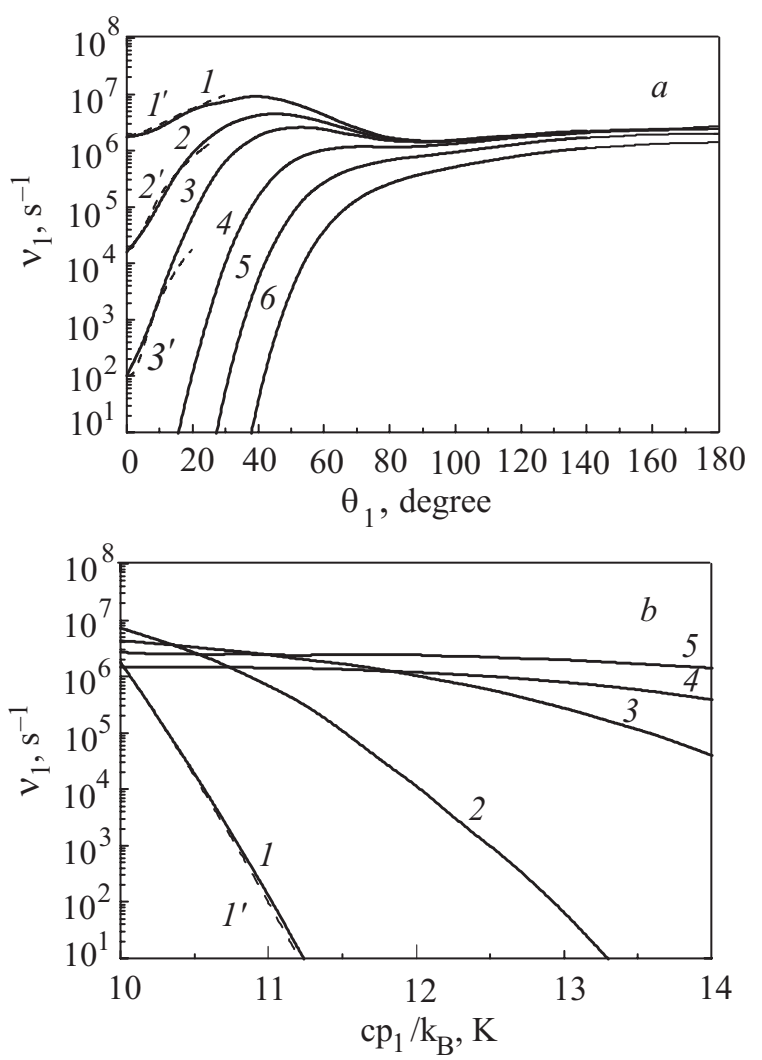

Fig. 1. $a-$ The rate $v_{1}$ is shown as a function of the angle $\theta_{1}$ between $\mathbf{p}_{1}$ and the propagation direction calculated from Eq. (38) for different values of $\widetilde{p}_{1}$ equal to 10 (1), 10.5 (2), 11 (3), 12 (4), 13 (5), and 14 (6) K. The dashed lines are corresponding approximate rates calculated from Eq. (62). $b$ - The dependence of the rate $v_{1}$ on momentum $c p_{1} / k_{B}$ calculated from Eq. (38) for different $\theta_{1}$ equal to $0^{\circ}(1), 30^{\circ}$ (2), $60^{\circ}(3), 90^{\circ}(4)$, and $180^{\circ}(5)$. The dashed lines are the corresponding approximate rates calculated from Eq. (62). All calculations had $T=0.041 \mathrm{~K}$ and $\chi=0.02$.

dependence of the rate $v_{1}$ at different values of $p_{1}$ and fixed values of $T=0.041 \mathrm{~K}$ and $\chi=0.02$, which correspond to the conditions of the experiments $[13,14]$. We see that there is a maximum when $\tilde{p}_{1}<13 \mathrm{~K}$. The appearance of this maximum at $\tilde{p}_{1}=10 \mathrm{~K}$ is caused by several reasons: firstly it follows from the conservation laws of energy and momentum that the four-phonon processes are prohibited if the angle between the momenta of phonons $\mathbf{p}_{1}$ and $\mathbf{p}_{2}$ is less than

$$
\zeta_{12 \min }=1-\cos \theta_{12 \min } \approx \frac{p_{1}+p_{2}}{p_{1} p_{2}} \phi
$$

Secondly the distribution function of phonons, in a pulse, has a sharp maximum at $\theta_{2}=0$. Thus, the majority of phonons in a pulse have momentum $\mathbf{p}_{2}$ directed along the anisotropy axis of the system. However at $\theta_{1}=0$ phonons with momentum $\mathbf{p}_{1}$ cannot interact with phonons having momentum $\mathbf{p}_{2}$ directed along the anisotropy axis because there is a minimum angle, $\theta_{12 \mathrm{~min}}$, for interaction between them. Thus, when $\theta_{1}=0$, the interaction of phonons with momentum $\mathbf{p}_{1}$ with most of the phonons of the pulse is prohibited by the conservation of energy and momentum. With increasing angle $\theta_{1}$, an increasing number of phonons in the pulse interact with the h-phonon. This leads to the increasing rate in the initial part of the curve, up to $\theta_{1} \sim 40^{\circ}$, see Fig. 1, $a$. The following decrease in the rate is caused by the decreasing value of the squared matrix element with increasing angle $\theta_{1}$. At these larger angles, the h-phonon with momentum $\mathbf{p}_{1}$ can interact with almost all phonons of the pulse. The squared matrix element is approximately constant for $\theta_{1} \geq \theta_{1 M E} \approx 80^{\circ}$ and even could be slightly increasing with the growth of $\theta_{1}$ in this range of angles. This is due to the cancellation of terms in (16). Then the slow growth of the rate at these angles is determined by the factor $R$ in the integrand of Eq. (38) and by the angular dependence of matrix element, see Fig 1,a. We note that it is important to retain all terms in $M_{\Sigma}$, if only one term is considered there are serious qualitative errors; for example for $M^{(1)}, M_{13}^{(3)}$ or $M_{14}^{(3)}$, the rate $v_{1}$ decreases with growth $\theta_{1}$, instead of increasing.

The situation is different when the $p_{1}$ is far from $p_{c}$. From (61) we see that angle $\theta_{12 \text { min }}$ increases with larger $p_{1}$ and becomes greater than the angle $\theta_{1 M E}$. Then the rate rises monotonically with increasing $\theta_{1}$ and there is no range of angles where the rate decreases. This behavior is apparent when $\tilde{p}_{1} \geq 13 \mathrm{~K}$, see Fig. 1, $a$.

In Fig. $1, b$ is shown the momentum dependence of the rate $v_{1}$ at different values of $\theta_{1}\left(0^{\circ}, 30^{\circ}, 60^{\circ}, 90^{\circ}\right.$ and $180^{\circ}$ corresponding to curves $1-5$, respectively) with the same values of $T$ and $\chi$ as in Fig. 1, $a$. It can be seen, that with increasing angle, the rate $v_{1}$ is higher in more of the momentum range, and at $\theta_{1}=180^{\circ}$ the dependence on momentum is actually absent. In Fig. $1, b$ we also see that the curves cross each other. The physical reasons for these behaviors are the same as those discussed above. We note that momentum dependence of $v_{1}$, when $\theta_{1}=0$, was ob-

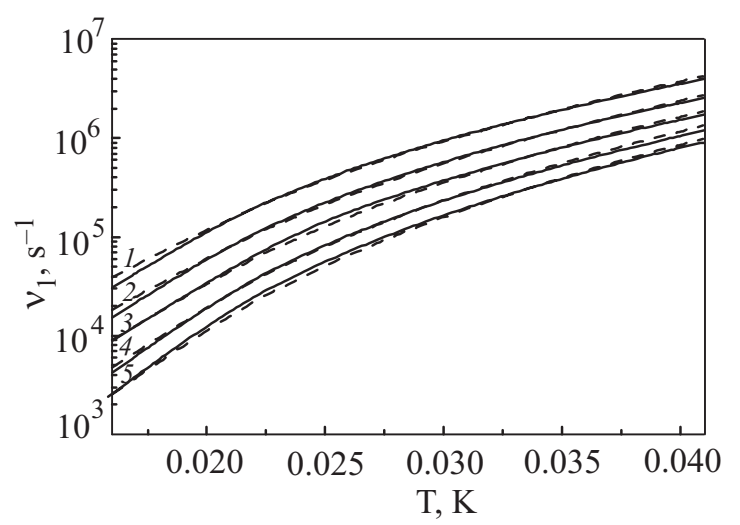

Fig. 2. The temperature dependence of the rate $v_{1}\left(p_{1}=p_{c}, \zeta_{1}=0\right)$ for different values of the anisotropy parameter $\chi$ equal to 0.01 (1), 0.015 (2), 0.02 (3), 0.025 (4), and 0.03 (5). The dashed lines represent the same dependences calculated from Eq. (62). 
tained in Ref. 9 with the approximate distribution function (10); the rate is similar to that presented here.

In Fig. 2 the dependence of $v_{1}$ on $T$ for different values of $\chi$ is shown for $\widetilde{p}_{1}=10 \mathrm{~K}$ and $\theta_{1}=0$. From Fig. 2 it can be seen, that the rate increases with both increasing temperature and with decreasing values of the anisotropy parameter $\chi$. Such behavior is completely determined by the dependence of distribution function $n_{2}^{(0)}$ on the parameters $\chi$ and $T$.

To solve a number of problems it is convenient to use analytical expression for $v_{1}$. However it is impossible to obtain an analytical expression for $v_{1}\left(p_{1}, \zeta_{1}, \chi, T\right)$ from the fivefold integral (38). In this case it is useful to have an approximate expression for $v_{1}\left(p_{1}, \zeta_{1}, \chi, T\right)$.

Using the data from the numerical calculation of the dependences of the rate on $p_{1}$ and $\zeta_{1}$, which was made with a help of Eq. (38) at different values of $\chi$ and $T$, then least squares fitting and taking into account (57), it is possible to obtain a numerical approximation for the rate $v_{1}$ on all parameters of the problem, which can be written as

$$
\begin{aligned}
& v_{1}\left(p_{1}, \zeta_{1}, \chi, T\right)=1.39 \cdot 10^{22} \cdot T^{\alpha_{1}\left(p_{1}\right)} \chi^{-\alpha_{2}\left(p_{1}\right)} \times \\
& \times \mathrm{e}^{\left(10 \frac{\psi_{1}}{T}-24.22\right) \frac{p_{1}}{p_{c}}+\alpha_{3}\left(p_{1}\right) \frac{\chi}{T}}\left(1+\alpha_{4}\left(p_{1}\right) \zeta_{1}^{\alpha_{5}\left(p_{1}\right)}\right),
\end{aligned}
$$

where $T$ should be substituted in Kelvins and $\alpha_{i}$ should be written as

$$
\begin{gathered}
\alpha_{1}\left(p_{1}\right)=10.37 \frac{p_{1}}{p_{c}}-6.06, \alpha_{2}\left(p_{1}\right)=3.11 \frac{p_{1}}{p_{c}}-2.53, \\
\alpha_{3}\left(p_{1}\right)=16.778 \frac{p_{1}}{p_{c}}-18.456, \\
\alpha_{4}\left(p_{1}\right)=24 \exp \left(29.95\left(\frac{p_{1}-p_{c}}{p_{c}}\right)^{0.668}\right), \\
\alpha_{5}\left(p_{1}\right)=6.62 \frac{p_{1}}{p_{c}}-5.71 .
\end{gathered}
$$

The approximation (62) can be used for $0.01 \leq \chi \leq 0.06$ and $0.016 \mathrm{~K} \leq T \leq 0.06 \mathrm{~K}$. We note that all experiments were carried out in this range of values of $\chi$ and $T$. Equation (62) is valid in the momentum range of $\tilde{p}_{1}$ from $\tilde{p}_{c}$ up to $11 \mathrm{~K}$, where the rate $N_{b}^{(\mathrm{sh})}$ is significantly above zero. We notice, that approximation (62) is applicable in the range of angles where $N_{b}^{(\text {sh })}$ is large. This is due to the fact that $v_{1}$ in $N_{b}^{(\text {sh })}$ is multiplied by $\exp \left[-\left(\varepsilon_{1}-\mathbf{p}_{1} \mathbf{u}\right) / k_{B} T\right]$, which very quickly decreases with increasing angle, and makes $N_{b}^{\text {(sh) }}$ relatively small at angles $\theta_{1} \geq 10^{\circ}$, see Fig. 3, $a$. The comparison of the numerical calculations of the rate with the approximation (62) one can see in Figs. 1 and 2 where the dashed curves were obtained with a help of Eq. (62). We see that for the given range of variables, the approximation (62) is rather good.
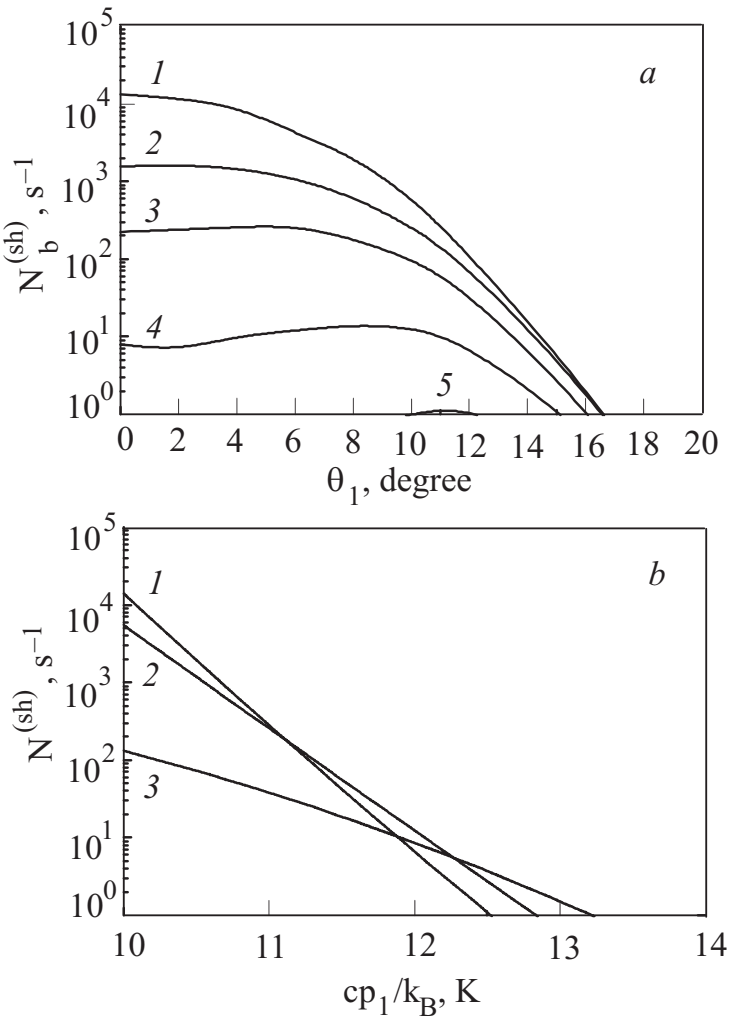

Fig. 3. $a$ - The creation rate of h-phonons, $N_{b}^{(\mathrm{sh})}$ in short pulses, is shown as a function of the angle $\theta_{1}$ between $\mathbf{p}_{1}$ and the propagation direction for different values of $c p_{1} / k_{B}$ equal to 10 (1), 10.5 (2), 11 (3), 12 (4) and 13 (5) K. $b$ - The dependence of $N_{b}^{(\mathrm{sh})}$ on momentum $c p_{1} / k_{B}$ for different values of $\theta_{1}$ equal to $0^{\circ}(1), 6^{\circ}(2)$, and $12^{\circ}(3)$. All curves have $\chi=0.02$ and $T=0.041 \mathrm{~K}$.

\section{Angular distribution of l- and h-phonons}

The angular distribution of 1- and h-phonons can be described by the probability density of the angular distribution of phonons. The probability density of created h-phonons as a function of $\zeta_{1}$ is defined by expression

$$
W_{h}\left(\zeta_{1}, \chi, T\right)=\frac{\int_{p_{c}}^{p_{\max }} \varepsilon_{1} N_{b}^{(\mathrm{sh})}\left(p_{1}, \zeta_{1}, \chi, T\right) p_{1}^{2} d p_{1}}{4 \pi^{2} \hbar^{3} \dot{E}_{h}},
$$

where

$$
\dot{E}_{h}=\frac{1}{4 \pi^{2} \hbar^{3}} \int_{0}^{2} d \zeta_{1} \int_{p_{c}}^{p_{\max }} \varepsilon_{1} N_{b}^{(\mathrm{sh})}\left(p_{1}, \zeta_{1}, \chi, T\right) p_{1}^{2} d p_{1}
$$

is the total energy of h-phonons, created in unit time and unit volume, $N_{b}^{\text {(sh) }}\left(p_{1}, \zeta_{1}, \chi, T\right)$ is defined by Eq. (25), and the upper limit of integration over momentum is chosen to be $p_{\max }=1.4 p_{c}$. The value of the integrals are not sensitive to the exact value of this upper limit as long as it is well above $p_{c}$ : even if $p_{\max }=1.1 p_{c}$ then the results 
change very little. The main contribution to the integrals is due to h-phonons with momentum close to $p_{c}$ because the function $N_{b}^{(\mathrm{sh})}\left(p_{1}\right)$ rapidly decreases when $p_{1}$ increases, see Fig. 3,b.

Similarly we can also introduce the probability density of 1-phonons as a function of $\zeta_{l}$

$$
W_{l}\left(\zeta_{l}, \chi, T\right)=\frac{\int_{0}^{p_{c}} \varepsilon_{l} n_{l}^{(0)}\left(p_{l}, \zeta_{l}, \chi, T\right) p_{l}^{2} d p_{l}}{4 \pi^{2} \hbar^{3} E_{l}},
$$

where

$$
E_{l}=\frac{1}{4 \pi^{2} \hbar^{3}} \int_{0}^{2} d \zeta_{l} \int_{0}^{p_{c}} \varepsilon_{l} n_{l}^{(0)}\left(p_{l}, \zeta_{l}, \chi, T\right) p_{l}^{2} d p_{l}
$$

is the total energy of 1-phonons in unit volume of the pulse, and $n_{l}^{(0)}\left(p_{l}, \zeta_{l}, \chi, T\right)$ is defined by Eq. (9).

The results of numerical calculations of the dependence of $W_{l}$ and $W_{h}$ on $\theta_{l, h}=\arccos \left(1-\zeta_{l, h}\right)$, obtained from Eqs. (67) and (69) at $\chi=0.02$ and $T=0.041 \mathrm{~K}$, which are values for $t=0$, are given in Fig. 4 . We see that $W_{h}$ is a considerably sharper function angle than $W_{l}$. For $\chi=0.061$ and $T=0.058 \mathrm{~K}$, which are values after $42 \mu$ s, we see that $W_{l}(t=42 \mu \mathrm{s})$ is even wider than $W_{l}(t=0)$.

The sharpness of functions $W_{l, h}$ is defined by the angular width of the corresponding distributions which are given by relation

$$
\bar{\zeta}_{l, h}=\int_{0}^{2} \zeta_{l, h} W_{l, h} d \zeta_{l, h}
$$

Figure 5 shows the dependences of $\bar{\theta}_{l, h}=\arccos \left(1-\bar{\zeta}_{l, h}\right)$ on $T$, at different values of $\chi$. It can be seen that the inequality $\bar{\theta}_{h}<\bar{\theta}_{l}$ is always satisfied. Thus the created

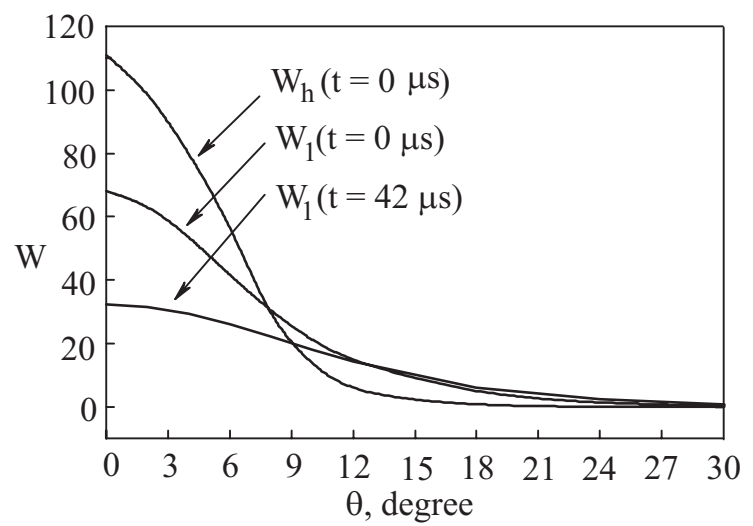

Fig. 4. The angular dependence of the probability density $W(t=0)$ for the $\mathrm{h}$ - and 1-phonon distributions at $\chi=0.02$ and $T=0.041 \mathrm{~K}$ obtained from Eqs. (67) and (69), respectively. Also $W_{l}(t=42 \mu \mathrm{s})$ is shown for $\chi=0.061$ and $T=0.058 \mathrm{~K}$ which are the corresponding values at $t=42 \mu \mathrm{s}$. Note the angular distribution is narrower for h-phonons than for 1-phonons.
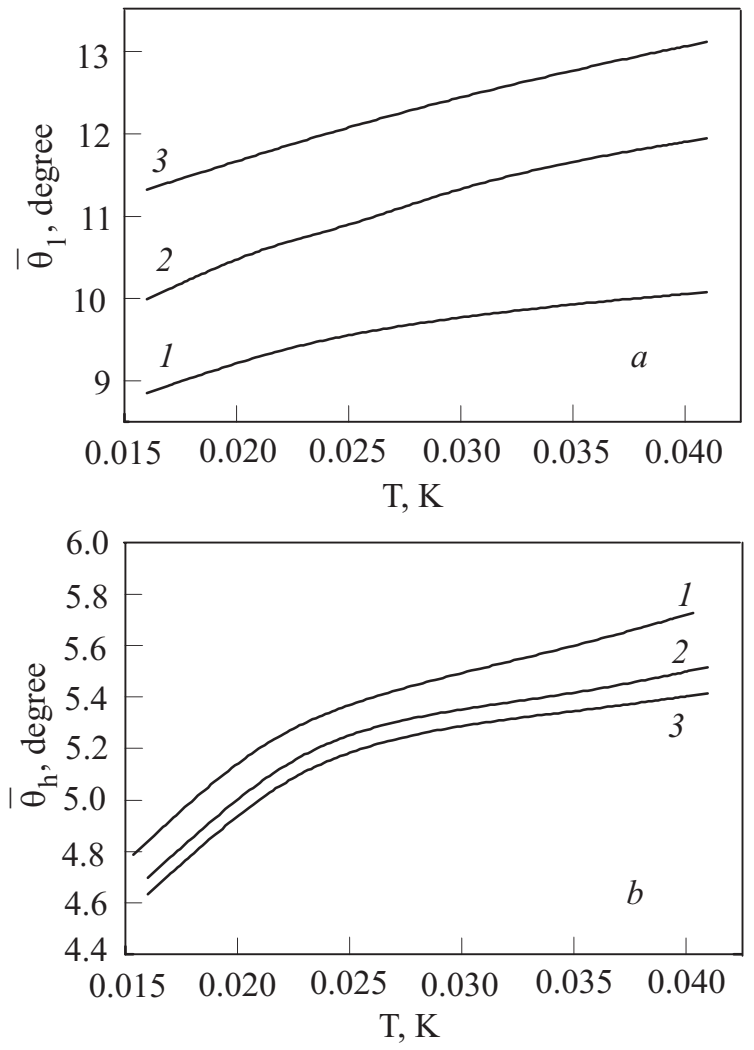

Fig. 5. The angular widths of the 1- and h-phonon distributions, $\bar{\theta}_{l}(a)$ and $\bar{\theta}_{h}(b)$, are shown as functions of temperature $T$, for different values of $\chi$ equal to 0.01 (1), 0.02 (2), and 0.03 (3).

h-phonons always occupy a narrower cone than the 1-phonons. This characteristic was observed in experiments [5] and was called «the concentration of h-phonons near the anisotropy axis of the system». Experiments showed, that h-phonons are in a cone with a cone angle about $4^{\circ}$, while 1-phonons occupy a cone with a cone angle close to $12^{\circ}$. We notice, that results of calculations with values of parameters which are typical for experiments $[13,14], T=0.041 \mathrm{~K}$ and $\chi=0.02$, give $\bar{\theta}_{l} \approx 12^{\circ}$ and $\bar{\theta}_{h} \approx 5.5^{\circ}$ near the heater, and near the bolometer we have $\bar{\theta}_{l}(t=42 \mu \mathrm{s}) \approx 16.8^{\circ}$ and $\theta_{h}$ is not expected to change much. These calculated values after $42 \mu$ s are similar to experimental values. Thus, the reasons for the observed concentration of h-phonons near the anisotropy axis of the system, can now be explained as follows.

The rapidly decreasing value of $W_{h}$ with increasing $\zeta_{1}$, is related to the angular dependence of $N_{b}^{(\mathrm{sh})}$ which is shown on Fig. 3. It can be seen in Fig. 3, $a$, that $N_{b}^{(\text {sh })}$ very quickly decreases with increasing $\theta_{1}$. This decrease is determined by the multiplier $\exp \left[-\left(\varepsilon_{1}-\mathbf{p}_{1} \mathbf{u}\right) / k_{B} T\right]$ in expression (25), which has a sharp maximum at $\theta_{1}=0$, see Eq. (26). While in Fig. 3, $a$ the presence of the exponential multiplier leads to the fast decrease of the function $N_{b}^{(\mathrm{sh})}\left(\theta_{1}\right)$ while function $v_{1}\left(\theta_{1}\right)$ is increasing. In Fig. 3,b the presence of the exponential multiplier makes the momentum dependence of $N_{b}^{(\mathrm{sh})}\left(p_{1}\right)$ more flat than the de- 


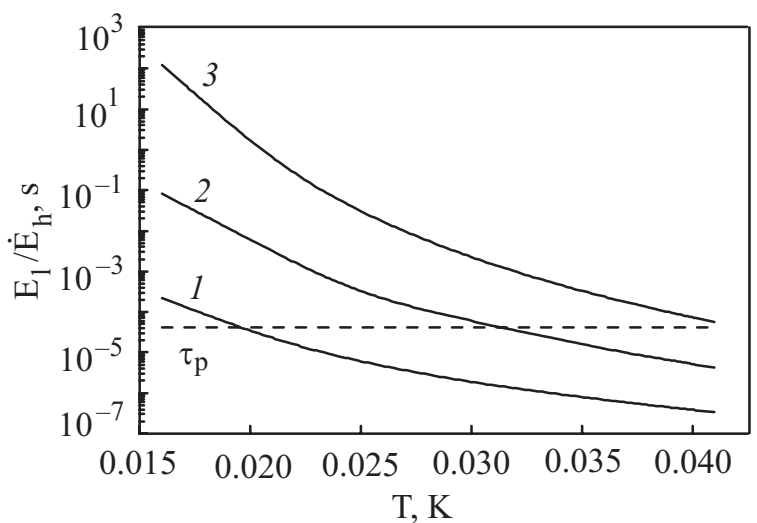

Fig. 6. The characteristic time for 1-phonon energy to be converted to h-phonon energy is given by the ratio $E_{l} / \dot{E}_{h}$ is shown as a function of temperature $T$ for different values of $\chi$ equal to 0.01 (1), 0.02 (2), and 0.03 (3). The dashed line shows the time for a pulse to travel $10 \mathrm{~mm}$, i.e. $42 \mu \mathrm{s}$, from Eq. (72). We see more energy is converted when $\chi$ is small, i.e., most anisotropic.

pendence $v_{1}\left(p_{1}\right)$. This can be explained by the fact that the exponential $\exp \left[-\left(\varepsilon_{1}-\mathbf{p}_{1} \mathbf{u}\right) / k_{B} T\right]$ quickly increases with increasing momentum $p_{1}$, at fixed values of $\theta_{1}$, due to the increase in the absolute value of the negative function $\psi\left(p_{1}\right)$ with increasing $p_{1}$, see Eq. (26).

An important characteristic of h-phonon creation is the relation $E_{l} / \dot{E}_{h}$. It is the characteristic time it takes for the energy of the created h-phonons to become equal to the initial energy of 1-phonon pulse, if the parameters $\chi$ and $T$ of 1-phonon pulse are assumed constant. This characteristic time shows the intensity of the h-phonon creation process.

Comparing this time with the time of the pulse propagation

$$
\tau_{p}=\frac{10 \mathrm{~mm}}{238 \mathrm{~m} / \mathrm{s}} \approx 4.2 \cdot 10^{-5} \mathrm{~s}=42 \mu \mathrm{s},
$$

we can determine whether 1-phonons have time to transfer a significant part of their energy to h-phonons during their propagation from the heater to the detector.

Figure 6 shows the dependence of $E_{l} / \dot{E}_{h}$ on temperature $T$ for different values of $\chi$. One can see that when $\chi=0.01$, the h-phonon creation is rapid at temperatures $T>0.02 \mathrm{~K}$, when $\chi=0.02$ at $T>0.032 \mathrm{~K}$, and when $\chi=0.03$ there is practically no h-phonon creation at any temperature.

\section{Evolution of a short l-phonon pulse caused by h-phonon creation}

A short phonon pulse is such that all the created h-phonons are lost from the 1-phonon pulse well before equilibrium between 1 - and $\mathrm{h}$-phonons is established. The kinetic equation (4) can be written as

$$
\frac{d n_{1}}{d t}=N_{b}^{(\mathrm{sh})} .
$$

We multiply the right and left part of Eq. (73) by $\varepsilon_{1}$ and integrate over all $d^{3} p_{1} /(2 \pi \hbar)^{3}$. As a result we have

$$
\frac{d \varepsilon_{h}}{d t}=\dot{E}_{h},
$$

where $\dot{E}_{h}$ is defined by expression (68), and

$$
\varepsilon_{h}=\frac{1}{4 \pi^{2} \hbar^{3}} \int_{0}^{2} d \zeta_{1} \int_{p_{c}}^{p_{\max }} d p_{1} p_{1}^{2} \varepsilon_{1} n_{1} .
$$

From conservation of energy it follows that

$$
\frac{d \varepsilon_{h}}{d t}=-\frac{d E_{l}}{d t},
$$

where $E_{l}$ is defined by expression (70). From (74) and (76) we have

$$
-\frac{d E_{l}}{d t}=\dot{E}_{h} .
$$

Now we multiply the right and left part of Eq. (73) by $p_{1 z}$ and in a similar way obtain

$$
-\frac{d P_{l}}{d t}=\dot{P}_{h},
$$

where

$$
\begin{gathered}
P_{l}=\frac{1}{4 \pi^{2} \hbar^{3}} \int_{0}^{2} d \zeta_{l} \int_{0}^{p_{c}} n_{l}^{(0)}\left(p_{l}, \zeta_{l}, \chi, T\right) p_{l}^{3}\left(1-\zeta_{l}\right) d p_{l}, \\
\dot{P}_{h}=\frac{1}{4 \pi^{2} \hbar^{3}} \int_{0}^{2} d \zeta_{1} \int_{p_{c}}^{p_{\max }} N_{b}^{(\mathrm{sh})}\left(p_{1}, \zeta_{1}, \chi, T\right) p_{1}^{3}\left(1-\zeta_{1}\right) d p_{1} .
\end{gathered}
$$

Thus, we have obtained two equations (77) and (78) that must be solved with respect to functions $\chi(t)$ and $T(t)$ with the initial conditions $\chi(t=0)=\chi_{0}$ and $T(t=0)=T_{0}$. The solution of combined equations (77) and (78) is discussed in detail in the Appendix B.

Figure 7, $a$ represents the temperature $T(t)$ dependence on time calculated with $T_{0}=0.041$ and $\chi_{0}=0.02,0.025$, $0.03,0.035$ (curves $1-4$, respectively). In Fig. $7, b$ is shown the anisotropy parameter $\chi(t)$ as a function of time, for $\chi_{0}=0.02$ and $T_{0}=0.025,0.030,0.036,0.041$ (curves $1-4$, respectively). It can be seen from Fig. 7 that h-phonon creation leads not only to increasing $\chi$ but also to increasing $T$; nevertheless the total energy density of 1-phonon pulse decreases with time (see Fig. 8).

In Fig. 8 the time dependence of the 1-phonons energy density $E_{l}(t)=E_{l}(\chi(t), T(t))$ and the 1-phonon pulse angular width $\bar{\theta}_{l}(t)=\bar{\theta}_{l}(\chi(t), T(t))$ are shown. The broadening of a pulse with time, seen in Fig. 8, is due to the con- 

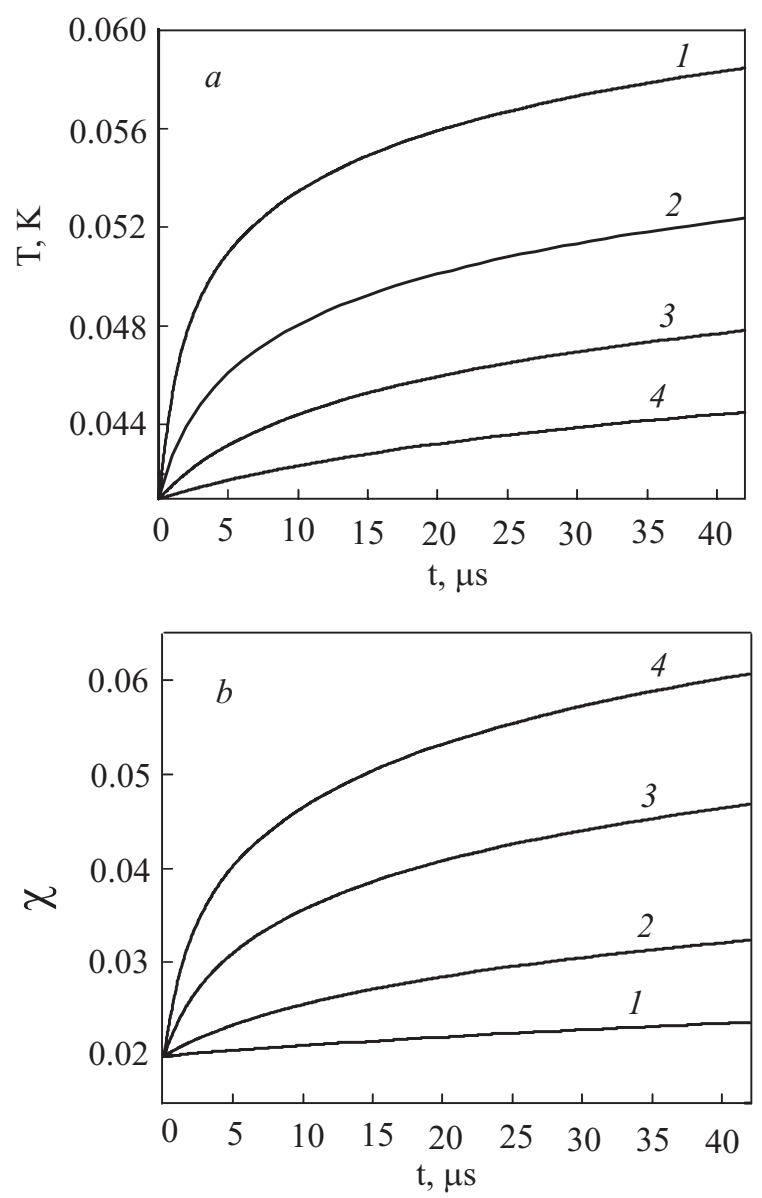

Fig. 7. $a$ - The temperature $T(t)$ is shown as a function of time, calculated with $T_{0}=0.041 \mathrm{~K}$ and $\chi_{0}=0.02(1), 0.025$ (2), 0.03 (3), 0.035 (4). $b$ - The anisotropy parameter $\chi(t)$ is shown as a function of time, calculated with $\chi_{0}=0.02$ and $T_{0}=0.025$ (1), 0.030 (2), 0.036 (3), 0.041 (4) K.

servation of momentum when h-phonons are created in the 1-phonon pulse. The created h-phonons, as can be seen in Fig. 4, have a sharper angular dependence and that is why they carry away momentum mainly parallel to the

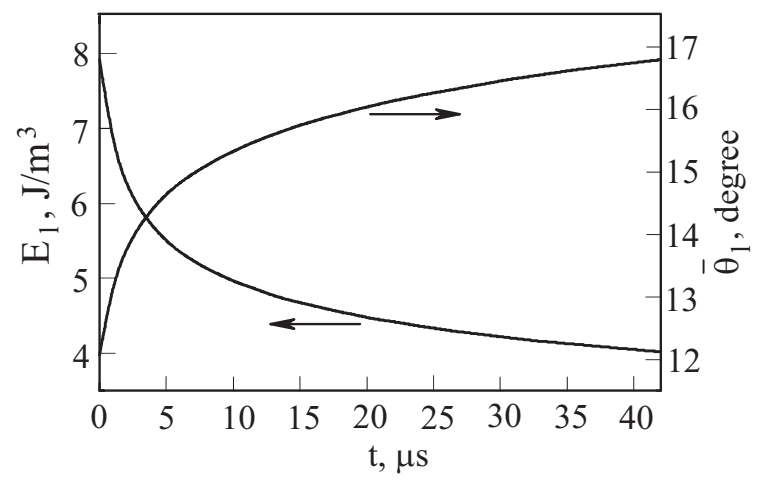

Fig. 8. The 1-phonon energy density $E_{l}(t)$ and the angular width of the 1-phonons in momentum space $\bar{\theta}_{l}(t)$ are shown as functions of propagation time, calculated with initial values of $\chi_{0}=0.02, T_{0}=0.041 \mathrm{~K}$.

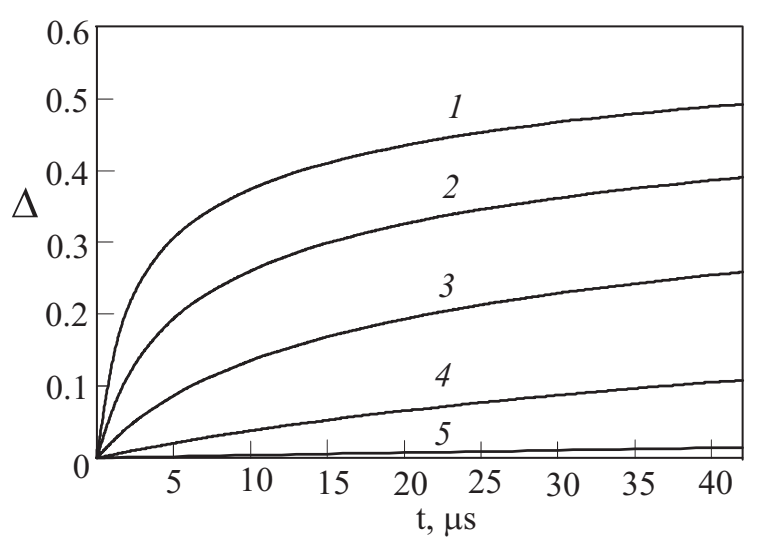

Fig. 9. The energy density lost by the 1-phonons, due to h-phonon creation, relative to the initial l-phonon energy density, $\Delta(t)$, is shown as a function of time calculated with $\chi_{0}=0.02$ for different values of $T_{0}$ equal to 0.041 (1), 0.036 (2), 0.031 (3), 0.026 (4), and 0.021 (5) K.

anisotropy axis. To compensate for this, the 1-phonon angular distribution must broaden.

For other values of $\chi_{0}$ and $T_{0}$, all the dependencies are qualitatively the same as shown in the figures.

We introduce

$$
\Delta(t)=\frac{E_{l}\left(\chi_{0}, T_{0}\right)-E_{l}(\chi(t), T(t))}{E_{l}\left(\chi_{0}, T_{0}\right)},
$$

which shows the fraction of the 1-phonon energy which transforms into h-phonons.

In Fig. 9 the function $\Delta(t)$ is shown at $\chi_{0}=0.02$ for five values of the initial temperature $T_{0}=0.041,0.036,0.031$, 0.026 , and $0.021 \mathrm{~K}$ (curves $1-5$, respectively). It can be seen from Fig. 9 that the process of h-phonon creation is more rapid at higher values of the initial temperature. The dependence $\Delta(t)$ shown in Fig. 9 is close to that obtained in $[7,8]$, which was derived with the approximate cone distribution function (10), and with corresponding values of $T_{p}$ and $\zeta_{p}$.

In Fig. 10, $a$ the dependences $E_{l}(t=0)$ (curve 1 ) and $E_{l}(t=42 \mu \mathrm{s})$ (curve 2 ) on the initial temperature $T_{0}$, with $\chi_{0}$ kept constant, are shown. We see, that with increasing of $T_{0}$, the energy density $E_{l}(t=42 \mu \mathrm{s})$ also increases. In Fig. 10, $b$ similar dependences of the angular widths $\bar{\theta}_{l}(t=0)$ (curve 1$)$ and $\bar{\theta}_{l}(t=42 \mu \mathrm{s})$ (curve 2 ) are shown. All the curves shown on Fig. 10 were calculated with the value of $\chi_{0}=0.02$ which is a typical measured value.

From Fig. 10 we see that with increasing initial temperature, we expect wider and higher energy pulses on the detector. Such behavior was observed in experiments when the initial power was increased, see Figs. 3 and 7 in Ref. 10. 

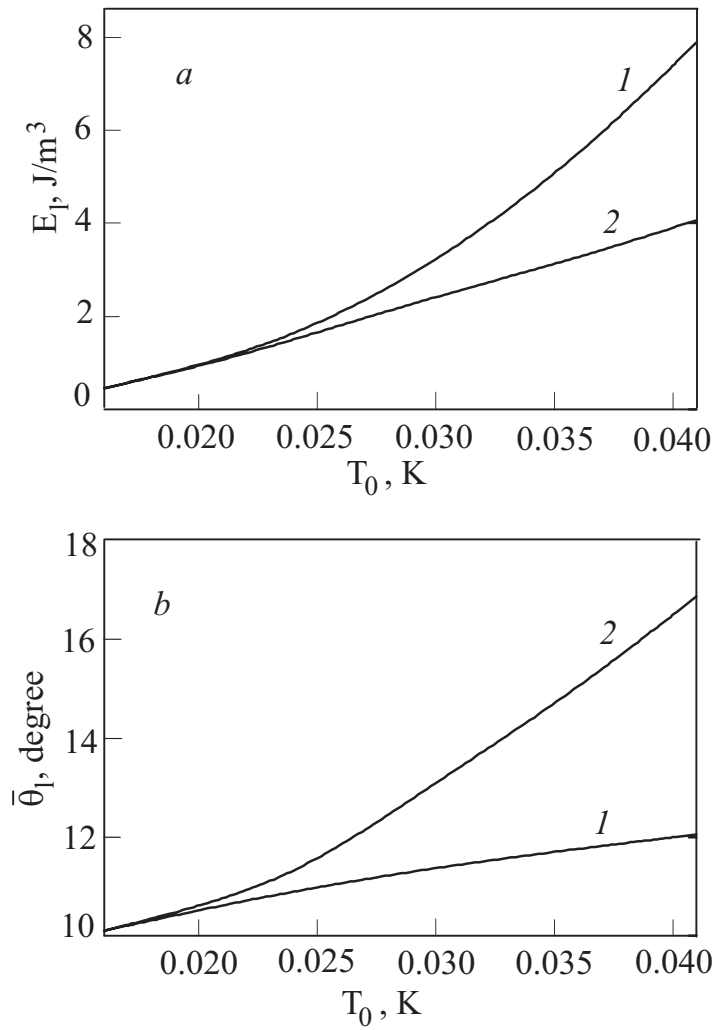

Fig. 10. $a-$ The initial 1-phonon energy density, $E_{l}(t=0)$ (curve 1$)$, and the energy density after $42 \mu \mathrm{s}, E_{l}(t=42 \mu \mathrm{s})$ (curve 2), are shown as functions of the initial temperature $T_{0}$. $b$ - The initial angular width of the 1-phonons in momentum space, $\bar{\theta}_{l}(t=0)$ (curve 1 ), and after $42 \mu \mathrm{s}, \bar{\theta}_{l}(t=42 \mu \mathrm{s})$ (curve $2)$, are shown as functions of the initial temperature $T_{0}$. All calculations were made with $\chi_{0}=0.02$.

\section{The momentum which separates the l- and h-phonon subsystems}

The calculations made above are based on the fact that phonons in superfluid helium separate into two subsystems according to their relaxation rate: 1-phonon subsystem in which equilibrium occurs quickly and h-phonon subsystem in which equilibrium occurs slowly. The basis for such division is the strong inequality (2). In this paper, as well as in previous ones (see Refs. 7, 8), it was supposed, that the momentum $p_{d}$ separating 1- and h-phonon subsystems is equal to $p_{c}$, after which the spontaneous decay of phonons is forbidden. However, according to Refs. 1, 19 the conservation laws of energy and momentum allow three-phonon processes $(1 \rightarrow 2)$ only up to momentum $p_{1 \rightarrow 2}=\sqrt{4 / 5} p_{c}$ (at the saturated vapour pressure $\tilde{p}_{1 \rightarrow 2}=8.94 \mathrm{~K}$ with our parametrization of the dispersion curve). In the momentum range from $p_{1 \rightarrow 2}$ to $p_{c}$ processes of one phonon decaying into more than two phonons are allowed. So, for example, one phonon decay into three is allowed by conservation laws up to momentum $p_{1 \rightarrow 3}=\sqrt{9 / 10} p_{c}\left(\tilde{p}_{1 \rightarrow 3}=9.49 \mathrm{~K}\right)$, into four - up to $9.7 \mathrm{~K}$, into five - up to $9.81 \mathrm{~K}$ and so on up to $\tilde{p}_{c}=10.0 \mathrm{~K}$.
At present there are no publications of calculations of the rates of the mentioned above processes, although 1 to 3 is submitted [20]. Hence there is the question of which subsystem phonons with momenta

$$
p_{1 \rightarrow 2} \leq p \leq p_{c}
$$

should be assigned. If the rates of one phonon decay into three, four, etc. appear much higher than the rate $v_{1}$ then these phonons should be part of the 1-phonon subsystem, and so $p_{d}=p_{c}$. Otherwise these phonons should be part of the h-phonon subsystem and in this case $p_{d}<p_{c}$.

The answer to this question is important as the numerical value of the rate $v_{1}$, for phonons moving at small angles to the anisotropy axis, is very sensitive to the value of $p_{d}$ when $p_{d}<p_{c}$. Such sensitivity is because the main contribution in Eq. (43) for $v_{1}$ is given by phonons with momentum $p_{3}$ close to the upper limit of integration $p_{d}$.

This reason for this is now explained. The distribution function $n_{2}^{(0)}$ exponentially decreases with increasing $\zeta_{2}$. As a result, the main contribution to the integration over $\zeta_{2}$ is given by phonons with $\zeta_{2}=\zeta_{12 \min }$ (see (61)). Hence the integration over $\zeta_{2}$ leads to the following factor in the integrand of (43)

$$
\exp \left(-\frac{c p_{2}}{k_{B} T} \zeta_{12 \min }\right) \approx \exp \left(-\frac{c \phi}{k_{B} T}\right),
$$

which for values of $T$ expected in experiments, quickly increases with decreasing $\phi$.

It is not difficult to check that $\phi$, determined by Eq. (30), monotonically decreases with increasing $p_{3}$ and is minimal (and the exponent function is accordingly maximal) when $p_{3}=p_{d}$. So if $T=0.041 \mathrm{~K}, p_{d}=p_{c}$ then for $\tilde{p}_{1}=10.001 \mathrm{~K}$ and the typical value of $\tilde{p}_{2}=1.58 \mathrm{~K}$ when $\widetilde{p}_{3}=10 \mathrm{~K}$, the exponent of the exponential function $-c \phi / k_{B} T=-5.35 \cdot 10^{-3}$, and when $\tilde{p}_{3}=8.95 \mathrm{~K}$, we have $-c \phi / k_{B} T=-4.67$; i.e., the value of the exponential function increases by two orders of magnitude. We see that re-

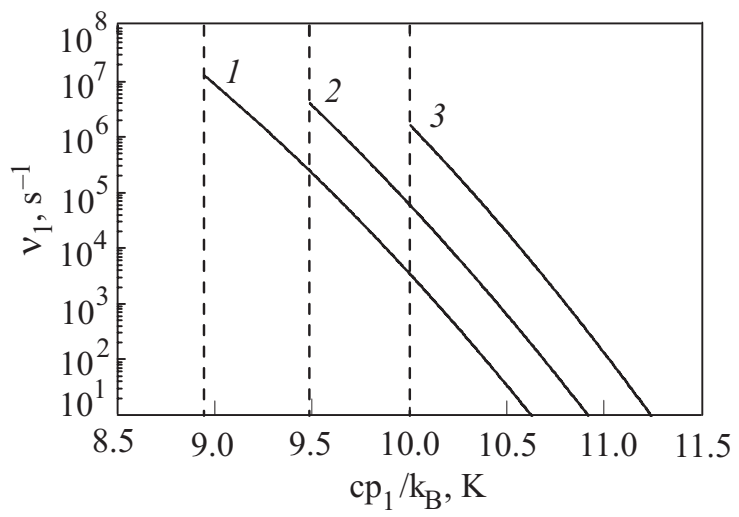

Fig. 11. The momentum dependence of the rate $v_{1}$ at $\theta_{1}=0$ for different values of $\tilde{p}_{d}$ equal to 8.94 (1), 9.49 (2), and 10 (3) $\mathrm{K}$. These dependences were obtained from Eq. (43) with $T=0.041 \mathrm{~K}$ and $\chi=0.02$. 
ducing $p_{d}$, and correspondly reducing the top limit of integration over $p_{3}$, leads to an exponential decrease of the rate $v_{1}\left(\mathbf{p}_{1}\right)$, at small $\theta_{1}$.

In Fig. 11 the momentum dependence of the rate $v_{1}\left(p_{1}\right)$ is shown at three different values of $\tilde{p}_{d}$ equal to $8.94,9.49$, and $10 \mathrm{~K}$ (curves $1-3$, respectively) at $\theta_{1}=0$. These dependences were obtained from expression (43) at values of $T=0.041 \mathrm{~K}$ and $\chi=0.02$. From Fig. 11 one can see, that with decreasing $\widetilde{p}_{d}$ from 10 to $9.5 \mathrm{~K}$, the rate $v_{1}\left(\widetilde{p}_{1}=10 \mathrm{~K}\right)$ decreases an order of magnitude.

The physical causes for such a strong decrease are the strong anisotropy of 1-phonon subsystem and the conservation laws (3) which make process $2 \rightarrow 2$ forbidden for angles $\theta_{12}<\theta_{12 \min }$. Such a high sensitivity to the choice of $p_{d}$ is absent in isotropic phonon systems. So when $\chi=1$, the calculation of the rate with a help of formula (43), the rate $v_{1}$ in isotropic phonon system, only changes 1.5 times, when $p_{d}$ goes from $p_{c}$ to $p_{1 \rightarrow 2}$.

So in order to answer the question of the value of $p_{d}$ in superfluid helium, it is necessary to compare results of the theory presented in this paper with experimental data [21], where the shape and the arrival time of h-phonon signal created by l-phonon pulse, at the bolometer, were investigated. It follows from the Eq. (25), that the exponential factor in $N_{b}^{(\text {sh ) }}$, cuts off at large angles $\theta_{1}$ (see Fig. 3). In connection with this the rate $v_{1}$, which is in $N_{b}^{(\text {sh })}$ (see Eq. (25)) is of great interest especially at small values of $\theta_{1}$. We note that in the case of small $\theta_{1}$ at different values of $p_{d}$, the rate $v_{1}$ is described by curves similar to those in Fig. 11 for $\theta_{1}=0$. From Fig. 11 we see that changing $p_{d}$, shifts the curves $v_{1}\left(p_{1}\right)$ to lower momenta by amounts equal to the differences in the values of $p_{d}$. Therefore if $p_{d}=p_{c}$, the maximum h-phonon signal should be formed by phonons with momentum $\widetilde{p}_{1} \approx 10.0 \mathrm{~K}$ and phonons with $\widetilde{p}_{1}=8.95 \mathrm{~K}$ should be absent. When $p_{d}=p_{1 \rightarrow 2}$ the maximum h-phonon signal should be due to phonons with $\widetilde{p}_{1} \approx 8.95 \mathrm{~K}$ and phonons with momentum $\widetilde{p}_{1}=10 \mathrm{~K}$ should be practically absent.

The group velocity $v_{\mathrm{gr}}$ of phonons depends on momentum. From the data in Ref. 22, at the saturated vapour pressure, $v_{\mathrm{gr}}(\tilde{p}=8.95 \mathrm{~K})=207 \mathrm{~m} / \mathrm{s}$ and $v_{\mathrm{gr}}(\tilde{p}=10 \mathrm{~K})=$ $=189 \mathrm{~m} / \mathrm{s}$. Therefore the position of the maximum of the h-phonon signal, which in experiments $[6,21]$ is measured with good accuracy, allows us to understand which phonons form this maximum and accordingly the correct value of $p_{d}$. Experiments $[6,21]$ show that the maximum of the h-phonon signal moves with a speed of $186.4 \mathrm{~m} / \mathrm{s}$, which corresponds to a group velocity of phonons with $\widetilde{p}_{1}=10.15 \mathrm{~K}$.

Calculations similar to those presented in the previous section, with $p_{d}=p_{c}$, give the shape of h-phonon signal close to that observed in Refs. 6, 21, and are distinctly different from the shape with smaller values of $p_{d}$. From this section we see that in superfluid helium, the momentum $p_{d}$ separating the 1 - and h-phonon subsystems is equal to $\widetilde{p}_{c}=10.0 \mathrm{~K}$, as it was supposed in this paper and earlier ones, Refs. 7, 8.

\section{Conclusion}

The general expression (38) for the rate $v_{1}$ which describes the decay (22) and creation (24) of high-energy phonons in a pulse of low-energy phonons moving from a heater to the detector, was found. Also the exact analytical expression for the rate $v_{1}$ when $\theta_{1}=0$ (see (43)) was found. This expression also describes the relaxation in isotropic phonon systems, i.e., when $\chi=1$. From Eq. (43) we derive a simple and explicit analytical expression for the rate $v_{1}$ for the case when h-phonons move along the anisotropy axis $\left(\zeta_{1}=0\right)(\operatorname{see}(57))$. This allowed us to get an analytical approximation for $v_{1}$ in the most interesting range of angles $\zeta_{1}$ (see (62)).

Starting from Eq. (38), the rates were a numerically calculated. This allowed us to obtain the momentum and angular dependences of the rate $v_{1}$ (see Fig. 1). Also the dependences of the rate $v_{1}$ on the parameters of 1-phonon pulse were obtained (see Fig. 2). It was shown, that angular dependence of the rate $v_{1}$ has a maximum at $\widetilde{p}_{1}<13 \mathrm{~K}$ (see Fig. 1,a). The physical reasons of this maximum were given and the detailed analysis of such behavior of the rate $v_{1}$ was made.

The calculation of the rate $v_{1}$ allowed us to get the angular distribution of created h-phonons and show that they occupy a solid angle, in momentum space, which is much smaller than the solid angle for 1-phonons (see Figs. 4 and 5). This result explains the observation that the created h-phonon are concentrated near the anisotropy axis of the system [5].

Starting from the kinetic equation (73), the problem of the evolution of a short 1-phonon pulse, caused by the creation of h-phonons, was solved. For this, the principal consideration was the angular dependence of the collision integral $N_{b}^{(\mathrm{sh})}$ in (73), which quickly decreases with increasing angle (see Fig. 3). The physical reasons of such behavior were analyzed, and it was shown that it is connected with the exponential factor in $N_{b}^{(\mathrm{sh})}$ (see Eq. (25)).

We investigated how the h-phonon creation rate depended on the parameters of the 1-phonon pulse. It was shown that h-phonon creation not only increased the anisotropy parameter $\chi$ of 1-phonon pulse but also increased the temperature $T$ (see Fig. 7). However the energy density of 1-phonon pulse decreased as it must (see Fig. 8). For the conditions used in the experiments $[13,14]$, the theory showed that up to $50 \%$ of initial energy of 1-phonon pulse could be transferred into h-phonon creation (see Fig. 9).

Also the dependence of the energy $E_{l}$ and the angular width $\bar{\theta}_{l}$ of the 1-phonon pulse at detector, on the initial temperature $T_{0}$, were investigated (see Fig. 10). It was shown, that with increasing initial temperature there will 
be wider and higher energy density pulses at the detector. Such phenomenon were observed in experiments [10] when different heater powers were used.

It was shown that the rate $v_{1}$, for small angles $\theta_{1}$, strongly depends on momentum $p_{d}$ which separates the 1and h-phonon subsystems (see Fig. 11). From a comparison of the present theory with experimental data we see that $p_{d}=p_{c}$.

\section{Acknowledgments}

We express our gratitude to EPSRC of the UK (grant EP/C 523199/1) for support of this work.

\section{Appendix A.}

\section{Expressions for the matrix elements}

Making the dot products in (17), (18), (20) and taking into account the conservation laws of energy and momentum (3) we have

$$
\begin{aligned}
& M^{(1)}=\frac{\varepsilon_{1+2}}{\varepsilon_{1}+\varepsilon_{2}-\varepsilon_{1+2}}\left(2 u-\zeta_{12}+\frac{2-\zeta_{12}}{\sqrt{1-\frac{2 p_{1} p_{2} \zeta_{12}}{\left(p_{1}+p_{2}\right)^{2}}}}\right)\left(2 u-\zeta_{34}+\frac{2-\zeta_{34}}{\sqrt{1-\frac{2 p_{3} p_{4} \zeta_{34}}{\left(p_{3}+p_{4}\right)^{2}}}}\right) \\
& M^{(5)}=-\frac{\varepsilon_{1+2}}{\varepsilon_{1}+\varepsilon_{2}+\varepsilon_{1+2}}\left(2 u-\zeta_{12}-\frac{2-\zeta_{12}}{\sqrt{1-\frac{2 p_{1} p_{2} \zeta_{12}}{\left(p_{1}+p_{2}\right)^{2}}}}\right)\left(2 u-\zeta_{34}-\frac{2-\zeta_{34}}{\sqrt{1-\frac{2 p_{3} p_{4} \zeta_{34}}{\left(p_{3}+p_{4}\right)^{2}}}}\right) \text {, } \\
& M_{13}^{(3)}=\frac{\varepsilon_{1-3}}{\varepsilon_{1}-\varepsilon_{3}-\varepsilon_{1-3}}\left(2 u-\zeta_{13}+\frac{2-\zeta_{13}}{\sqrt{1+\frac{2 p_{1} p_{3} \zeta_{13}}{\left(p_{1}-p_{3}\right)^{2}}}}\right)\left(2 u-\zeta_{24}+\frac{2-\zeta_{24}}{\sqrt{1+\frac{2 p_{2} p_{4} \zeta_{24}}{\left(p_{2}-p_{4}\right)^{2}}}}\right) \text {, } \\
& M_{24}^{(3)}=-\frac{\varepsilon_{1-3}}{\varepsilon_{1}-\varepsilon_{3}+\varepsilon_{1-3}}\left(2 u-\zeta_{13}-\frac{2-\zeta_{13}}{\sqrt{1+\frac{2 p_{1} p_{3} \zeta_{13}}{\left(p_{1}-p_{3}\right)^{2}}}}\right)\left(2 u-\zeta_{24}-\frac{2-\zeta_{24}}{\sqrt{1+\frac{2 p_{2} p_{4} \zeta_{24}}{\left(p_{2}-p_{4}\right)^{2}}}}\right) \text {, } \\
& M_{14}^{(3)}=\frac{\varepsilon_{1-4}}{\varepsilon_{1}-\varepsilon_{4}-\varepsilon_{1-4}}\left(2 u-\zeta_{14}+\frac{2-\zeta_{14}}{\sqrt{1+\frac{2 p_{1} p_{4} \zeta_{14}}{\left(p_{1}-p_{4}\right)^{2}}}}\right)\left(2 u-\zeta_{23}+\frac{2-\zeta_{23}}{\sqrt{1+\frac{2 p_{2} p_{3} \zeta_{23}}{\left(p_{2}-p_{3}\right)^{2}}}}\right) \text {, } \\
& M_{23}^{(3)}=-\frac{\varepsilon_{1-4}}{\varepsilon_{1}-\varepsilon_{4}+\varepsilon_{1-4}}\left(2 u-\zeta_{14}-\frac{2-\zeta_{14}}{\sqrt{1+\frac{2 p_{1} p_{4} \zeta_{14}}{\left(p_{1}-p_{4}\right)^{2}}}}\right)\left(2 u-\zeta_{23}-\frac{2-\zeta_{23}}{\sqrt{1+\frac{2 p_{2} p_{3} \zeta_{23}}{\left(p_{2}-p_{3}\right)^{2}}}}\right) \text {, }
\end{aligned}
$$

where

$$
\zeta_{23}=\frac{\left(p_{1}-p_{4}\right)^{2}-\left(p_{2}-p_{3}\right)^{2}+2 p_{1} p_{4} \zeta_{14}}{2 p_{2} p_{3}}
$$

$$
\zeta_{34}=\frac{\left(p_{3}+p_{4}\right)^{2}-\left(p_{1}+p_{2}\right)^{2}+2 p_{1} p_{2} \zeta_{12}}{2 p_{3} p_{4}}
$$




$$
\begin{gathered}
\zeta_{24}=\frac{\left(p_{1}-p_{3}\right)^{2}-\left(p_{2}-p_{4}\right)^{2}+2 p_{1} p_{3} \zeta_{13}}{2 p_{2} p_{4}}, \\
\zeta_{1 i}=\zeta_{1}+\zeta_{i}-\zeta_{1} \zeta_{i}-\sqrt{2 \zeta_{1}-\zeta_{1}^{2}} \sqrt{2 \zeta_{i}-\zeta_{i}^{2}} \cos \left(\varphi_{i}-\varphi_{1}\right),
\end{gathered}
$$

\section{Appendix B. \\ Solution of combined equations (77) and (78)}

To solve combined equations (77) and (78) it is convenient to write them as

$$
\left\{\begin{array}{l}
-\frac{d E_{l}}{d t}=\dot{E}_{h}, \\
\frac{d}{d t}\left(E_{l}-c P_{l}\right)=-\left(\dot{E}_{h}-c \dot{P}_{h}\right) .
\end{array}\right.
$$

The system of equations (B.1) can be solved for $\dot{\chi}(t)$ and $\dot{T}(t)$. This system of two equations with initial conditions $\chi(t=0)=\chi_{0}$ and $T(t=0)=T_{0}$ can be solved numerically and the functions $\chi(t)$ and $T(t)$ found. However it is always convenient to carry out calculations with simple analytical expressions, which approximate the dependences of the functions in (94) on $\chi$ and $T$. Such expressions can be introduced as products of power functions and exponents. The form of these functions was obtained from (68), (70), (79), (80). The exponents of the power function and the coefficients in exponential functions, were calculated by a least squares fitting.

For the intervals corresponding to the conditions in experiments, $0.01 \leq \chi \leq 0.06$ and $0.016 \mathrm{~K} \leq T \leq 0.06 \mathrm{~K}$, approximations of the functions contained in (B.1) can be written in SI system of units as

$$
\begin{array}{r}
E_{l}(\chi, T)=3713.36 \mathrm{e}^{-0.503 \frac{\chi}{T}+0.0135 \frac{1}{T}} \chi^{-1.07} T^{3.25},(\mathrm{~B} .2) \\
E_{l}(\chi, T)-c P_{l}(\chi, T)=445.856 \mathrm{e}^{-0.435 \frac{\chi}{T}-0.00112 \frac{1}{T}} \chi^{-0.785} T^{3.24},
\end{array}
$$

$$
\begin{gathered}
\dot{E}_{h}(\chi, T)=8.51 \cdot 10^{13} \mathrm{e}^{-11.35 \frac{\chi}{T}-0.00648 \frac{1}{T}} \chi^{-0.831} T^{4.75}, \text { (B.4) } \\
\dot{E}_{h}(\chi, T)-c \dot{P}_{h}(\chi, T)=2.59 \cdot 10^{12} \mathrm{e}^{-11.18 \frac{\chi}{T}+0.0158 \frac{1}{T}} \chi^{-1.15} T^{5.96} .
\end{gathered}
$$

Having substituted (B.2)-(B.5) into (B.1) and made the differentiation, we obtain the system of two equations with respect to $\dot{\chi}$ and $\dot{T}$. The numerical solution of this system gives $\chi\left(t, \chi_{0}, T_{0}\right)$ and $T\left(t, \chi_{0}, T_{0}\right)$.

1. I.N. Adamenko, Yu.A. Kitsenko, K.E. Nemchenko, V.A. Slipko, and A.F.G. Wyatt, Low Temp. Phys. 31, 459 (2005) [Fiz. Nizk. Temp. 31, 607 (2005)].

2. I.N. Adamenko, Yu.A. Kitsenko, K.E. Nemchenko, V.A. Slipko, and A.F. G. Wyatt, Phys. Rev. B72, 054507 (2005).

3. I.N. Adamenko, K.E. Nemchenko, V.A. Slipko, and A.F.G. Wyatt, J. Phys.: Condens. Matter 17, 1 (2005).

4. A.F.G. Wyatt, N.A. Lockerbie, and R.A. Sherlock, J. Phys.: Condens. Matter 1, 3507 (1989).

5. M.A.H. Tucker and A.F.G. Wyatt, J. Phys.: Condens. Matter 6, 2813 (1994).

6. M.A.H. Tucker and A.F.G. Wyatt, J. Low Temp. Phys. 113, 621 (1998).

7. I.N. Adamenko, K.E. Nemchenko, A.V. Zhukov, M.A.H Tucker, and A.F.G. Wyatt, Phys. Rev. Lett. 82, 1482 (1999).

8. A.F.G. Wyatt, M.A.H. Tucker, I.N. Adamenko, K.E. Nemchenko, and A.V. Zhukov, Phys. Rev. B62, 9402 (2000).

9. I.N. Adamenko, K.E. Nemchenko, and A.F.G. Wyatt, J. Low Temp. Phys. 125, 1 (2001).

10. R. Vovk, C.D.H. Williams, and A.F.G. Wyatt, Phys. Rev. B68, 134508 (2003).

11. I.N. Adamenko, Yu.A. Kitsenko, K.E. Nemchenko, V.A. Slipko, and A.F.G. Wyatt, Phys. Rev. B73, 134505 (2006).

12. I.N. Adamenko, K.E. Nemchenko, and A.F.G. Wyatt, J. Low Temp. Phys. 126, 1471 (2002).

13. D.H.S. Smith, R. Vovk, C.D.H. Williams, and A.F.G. Wyatt, Phys. Rev. B72, 054506 (2005).

14. R. Vovk, C.D.H. Williams, and A.F.G. Wyatt, Phys. Rev. Lett. 91, 235302 (2003).

15. I.M. Khalatnikov, An Introduction to the Theory of Superfluidity, Addison-Wesley, Redwood City, CA (1989).

16. M.A.H. Tucker and A.F.G. Wyatt, J. Phys.: Condens. Matter 4, 7745 (1992).

17. V.M. Apalkov and M.E. Portnoi, Phys. Rev. B65, 125310 (2002).

18. V.M. Apalkov and M.E. Portnoi, Phys. Rev. B66, 121303 (2002).

19. J. Jackle and K.W. Kehr, Phys. Rev. Lett. 27, 654 (1971).

20. I.N. Adamenko, Yu.A. Kitsenko, K.E. Nemchenko, V.A. Slipko, and A.F.G. Wyatt, The Process of One Phonon Decaying into Three Phonons in Superfluid Helium, submitted.

21. R.V. Vovk, C.D.H. Williams, and A.F.G.Wyatt, Phys. Rev. B69, 144524 (2004).

22. W.G. Stirling, in $75^{\text {th }}$ Jubilee Conference on Liquid Helium-4, J.G.M. Armitage (ed.), World Scientic, Singapore (1983), p. 109. 